\title{
Randomized observation periods for compound Poisson risk model with capital injection and barrier dividend
}

\author{
Wenguang Yu ${ }^{1 *} \mathbb{D}$, Peng Guo², Qi Wang ${ }^{3}$, Guofeng Guan ${ }^{3}$, Yujuan Huang ${ }^{4}$ and Xinliang Yu'
}

\section{"Correspondence:}

yuwg@sdufe.edu.cn

'School of Insurance, Shandong

University of Finance and

Economics, Jinan 250014, P.R. China

Full list of author information is

available at the end of the article

\section{Springer}

\begin{abstract}
In this paper, we model the insurance company's surplus by a compound Poisson risk model, where the surplus process can only be observed at random observation times. It is assumed that the insurer observes its surplus level periodically to decide on dividend payments and capital injection at the interobservation time having an Erlang $(n)$ distribution. If the observed surplus level is greater than zero but less than injection line $b_{1}>0$, the shareholders should immediately inject a certain amount of capital to bring the surplus level back to the injection line $b_{1}$. If the observed surplus level is larger than dividend line $b_{2}\left(b_{2}>b_{1}\right)$, any excess of the surplus over $b_{2}$ is immediately paid out as dividends to the shareholders of the company. Ruin is declared when the observed surplus level is negative. We derive the explicit expressions of the Gerber-Shiu function, the expected discounted capital injection, and the expected discounted dividend payments. Numerical illustrations are also given to analyze the effect of random observation times on actuarial quantities.
\end{abstract}

MSC: 39A60; 91B30

Keywords: Compound Poisson risk model; Capital injection; Barrier dividend strategy; Gerber-Shiu function; The expected discounted capital injection; The expected discounted dividend payments; Randomized observation; Laplace transform

\section{Introduction}

Following the classical risk model introduced by Lundberg [1], we suppose that the surplus process of an insurance company, denoted by $\{X(t)\}_{t \geq 0}$, follows the classical CramérLundberg process,

$$
X(t)=u+c t-S(t)=u+c t-\sum_{k=1}^{N(t)} Y_{k}, \quad t \geq 0
$$

where $u \geq 0$ is an initial surplus, or capital, $c>0$ is the constant premium income per unit time. The aggregate insurance claim process $S(t)=\sum_{k=1}^{N(t)} Y_{k}$ is a compound Poisson process, where $N(t)$ is a Poisson process with intensity parameter $\lambda$, which represents the

(c) The Author(s) 2021. This article is licensed under a Creative Commons Attribution 4.0 International License, which permits use, sharing, adaptation, distribution and reproduction in any medium or format, as long as you give appropriate credit to the original author(s) and the source, provide a link to the Creative Commons licence, and indicate if changes were made. The images or other third party material in this article are included in the article's Creative Commons licence, unless indicated otherwise in a credit line to the material. If material is not included in the article's Creative Commons licence and your intended use is not permitted by statutory regulation or exceeds the permitted use, you will need to obtain permission directly from the copyright holder. To view a copy of this licence, visit http://creativecommons.org/licenses/by/4.0/. 
number of claims up to time $t ;\left\{Y_{k}\right\}_{k \geq 1}$ is a sequence of positive, independent, and identically distributed random variables representing the claim amount. As usual, $\{N(t)\}_{t \geq 0}$ and $\left\{Y_{k}\right\}_{k \geq 1}$ are independent of each other.

At present, the classical insurance risk model and its extended forms, such as dividend strategy, capital injection strategy, investment strategy, reinsurance strategy, etc., have been studied by many scholars, interested readers may refer to Gerber and Shiu [2], Chi and Lin [3], Yu [4], Yin et al. [5], Shen et al. [6], Yu et al. [7, 8], Zhou et al. [9, 10], Xu et al. [11], Yin and Wen [12], Dong et al. [13], Li et al. [14], Peng and Wang [15], Yao et al. [16], He and Liang [17], and Zhu and Yang [18]. It should be stressed in particular that the above dividend and capital injection are all considered as continuous, but this is not consistent with the actual situation. In the actual economic activities, the board of directors of the company generally holds a meeting at certain periods of time, and then decides whether to pay dividends to shareholders or inject capital into the insurance company, which result in that dividend payments or capital injection occurs at some discrete time points rather than at continuous time points, so the periodic dividend strategy or periodic capital injection is more in line with the actual situation. Therefore, it is necessary to study this kind of risk model with randomized observation. Albrecher et al. [19] study a modification of the horizontal dividend barrier strategy by introducing random observation times at which dividends can be paid and ruin can be observed. Avanzi et al. [20] study a periodic dividend barrier strategy in the dual model with continuous monitoring of solvency. Zhao et al. [21] investigate an optimal periodic dividend and capital injection problem for spectrally positive Lévy processes, and both proportional and fixed transaction costs from capital injection are considered. Zhang et al. [22] and Cheung and Zhang [23] study periodic dividend threshold-type strategy under a compound Poisson risk model, in which the observation interval follows the Erlang distribution. Peng et al. [24] consider a perturbed compound Poisson model and suppose that the insurance company can only observe the surplus process and decide whether to pay dividends at some discrete time points. Pérez and Yamazaki [25] and Noba et al. [26] study the optimality of periodic barrier strategies for a spectrally positive Lévy process and Lévy risk processes, respectively. Other relevant literature can be found in Yang and Deng [27], Dong and Zhou [28], Dong and Zhao [29], Yang et al. [30], Liu et al. [31], and Yu et al. [32].

In this paper, we assume that the insurance company can only observe the surplus process at a series of discrete time points $\left\{Z_{k}\right\}_{k=1}^{\infty}$ (i.e., $Z_{k}$ is the $k$ th observation time, with $\left.Z_{0}=0\right)$. Let $T_{k}=Z_{k}-Z_{k-1}(k=1,2, \ldots)$ denote the $k$ th interobservation time and assume that $\left\{T_{k}\right\}_{k=1}^{\infty}$ is an i.i.d. sequence distributed as a generic random variable $T$ and independent of $\{N(t)\}_{t \geq 0}$ and $\left\{Y_{k}\right\}_{k \geq 1}$. Under the above discrete assumptions, we introduce the periodic dividend strategy and capital injection strategy into the classic risk model (1). At the time of observation $Z_{k}$, if the current surplus $u$ of the insurance company is less than zero, ruin occurs immediately. If the current surplus $u$ is greater than zero but less than injection line $b_{1}\left(b_{1}>0\right)$, the shareholders should immediately inject capital $b_{1}-u$ to bring the surplus level back to the injection line $b_{1}$. If the current surplus $u$ exceeds the dividend line $b_{2}\left(b_{2}>b_{1}\right)$, a lump sum dividend payments of size $u-b_{2}$ will be paid immediately. Ruin is declared when the observed surplus level is negative (see Fig. 1). In addition, we assume that no matter what the level of surplus is, ruin, capital injection, dividend payments, and other acts will not happen outside the observation time point. With the above-defined dividend and capital injection rules, denote the sequences of surplus 


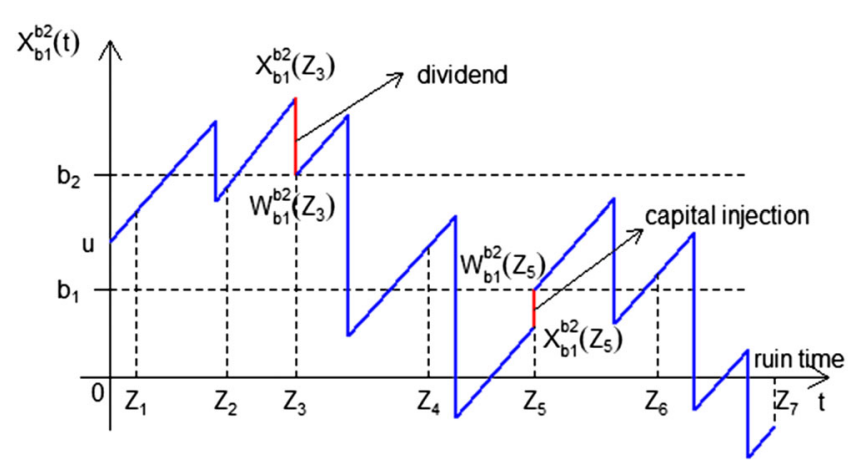

Figure 1 Sample path of $x_{b_{1}}^{b_{2}}(k)$

levels at the time points $\left\{Z_{k}^{-}\right\}_{k=1}^{\infty}$ and $\left\{Z_{k}\right\}_{k=1}^{\infty}$ by $\left\{X_{b_{1}}^{b_{2}}(k)\right\}_{k=0}^{\infty}$ and $\left\{W_{b_{1}}^{b_{2}}(k)\right\}_{k=0}^{\infty}$, respectively, i.e., $\left\{X_{b_{1}}^{b_{2}}(k)\right\}_{k=0}^{\infty}$ and $\left\{W_{b_{1}}^{b_{2}}(k)\right\}_{k=0}^{\infty}$ are the surplus levels at the $k$ th observation before (after, respectively) potential dividend payments or capital injection. With initial surplus level $X_{b_{1}}^{b_{2}}(0)=W_{b_{1}}^{b_{2}}(0)=u$, that is, at time zero, neither capital injection nor dividend payments are required, we then have the following surplus process of the modified risk model:

$$
\left\{\begin{array}{l}
X_{b_{1}}^{b_{2}}(k)=W_{b_{1}}^{b_{2}}(k-1)+X\left(Z_{k}\right)-X\left(Z_{k-1}\right), \quad k=1,2, \ldots \\
W_{b_{1}}^{b_{2}}(k)=\min \left[\max \left(X_{b_{1}}^{b_{2}}(k), b_{1}\right), b_{2}\right]
\end{array}\right.
$$

We then let $\tau_{b_{1}}^{b_{2}}$ be the ruin time defined as $\tau_{b_{1}}^{b_{2}}=Z_{k^{*}}$, where $k^{*}=\inf \left\{k \geq 1 \mid X_{b_{1}}^{b_{2}}(k)<\right.$ $0\}$. In this paper, we are interested in studying the Gerber-Shiu function, the expected discounted capital injection and the expected discounted dividend payments.

The Gerber-Shiu function is defined as follows:

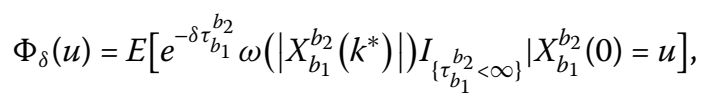

where $\delta$ is the force of interest, $I_{A}$ is the indicator function of the event $A$. The quantity $\omega(x)$ is a nonnegative measurable function defined on $[0, \infty)$ that can be interpreted as a penalty at the time of ruin for a deficit upon ruin of $\left|X_{b_{1}}^{b_{2}}\left(k^{*}\right)\right|$. In particular, if the function $\omega(x) \equiv 1$ and $\delta>0$, then $\Phi_{\delta}(u)=E\left[e^{-\delta \tau_{b_{1}}^{b_{2}}} I_{\left\{\tau_{b_{1}}^{b_{2}}<\infty\right\}} \mid X_{b_{1}}^{b_{2}}(0)=u\right]$ represents the Laplace transformation of the ruin time. The relevant references of Gerber-Shiu function can be found in Gerber and Shiu [33], Lin et al. [34], Willmot and Dickson [35], Li et al. [36], Huang et al. [37], Zhang and Su [38], Preischl and Thonhauser [39], Zhang et al. [40], and Palmowski and Vatamidou [41].

The expected discounted capital injection is described by

$$
\Psi(u)=E\left[\sum_{k=1}^{\infty} e^{-\delta Z_{k}} \chi_{1}\left(b_{1}-X_{b_{1}}^{b_{2}}(k)\right) I_{\left\{Z_{k}<\tau_{b_{1}} b_{2}\right\}} \mid X_{b_{1}}^{b_{2}}(0)=u\right],
$$

where the function $\chi_{1}(x)$ is a nonnegative function of the amount of capital injection for $x \in\left(0, b_{1}\right]$, and $\chi_{1}(x)=0$ for $x \leq 0$. 
The expected discounted dividend payments are defined as follows:

$$
\phi(u)=E\left[\sum_{k=1}^{\infty} e^{-\delta Z_{k}} \chi_{2}\left(X_{b_{1}}^{b_{2}}(k)-b_{2}\right) I_{\left\{Z_{k}<t_{b_{1}} b_{2}\right\}} \mid X_{b_{1}}^{b_{2}}(0)=u\right],
$$

where the function $\chi_{2}(x)$ is a nonnegative function of the amount of dividends payment for $x>0$, and $\chi_{2}(x)=0$ for $x \leq 0$.

In order to facilitate the description of the formula, we preprocess the model as follows. It is assumed that the interobservation time $T$ follows the $\operatorname{Erlang}(n, \gamma)$ distribution with density

$$
h_{T}(t)=\frac{\gamma^{n} t^{n-1} e^{-\gamma t}}{(n-1) !}, \quad t>0, \gamma>0
$$

and the claim amount $Y$ follows an arbitrary distribution on $(0,+\infty)$. The density function of $Y$ is $f_{Y}(y)$, the corresponding Laplace transformation is

$$
\tilde{f}_{Y}(s)=\int_{0}^{\infty} e^{-s y} f_{Y}(y) d y
$$

and assume that $\tilde{f}_{Y}(s)$ can be rewritten as follows:

$$
\tilde{f}_{Y}(s)=\frac{Q_{2, r-1}(s)}{Q_{1, r}(s)}
$$

where $Q_{1, r}(s)$ is a polynomial in $s$ of degree $r, Q_{2, r-1}(s)$ is a polynomial in $s$ of degree at most $r-1$. We also suppose that $Q_{1, r}(s)$ and $Q_{2, r-1}(s)$ have no common zeros, and $Q_{1, r}(s)$ has leading coefficient 1 . According to Albrecher et al. [42], the pairs $\left(T_{k}, X_{b_{1}}^{b_{2}}(k-1)-X_{b_{1}}^{b_{2}}(k)\right)$ $(k=1,2, \ldots)$ form an i.i.d. sequence with generic distribution $\left(T, \sum_{i=1}^{N(t)} Y_{i}-c T\right)$, and joint Laplace transform

$$
\begin{aligned}
E\left[e^{-\delta T-s\left(\sum_{i=1}^{N(t)} Y_{i}-c T\right)}\right] & =E\left[e^{-(\delta-c s) T} E\left[e^{-s \sum_{i=1}^{N(t)} Y_{i}} \mid T\right]\right] \\
& =E\left[e^{-\left\{(\delta-c s) T+\lambda\left[1-\tilde{f}_{Y}(s)\right] T\right\}}\right] \\
& =\left(\frac{\gamma}{\gamma+\delta-c s+\lambda\left[1-\tilde{f}_{Y}(s)\right]}\right)^{n} .
\end{aligned}
$$

In addition, the above formula can be changed into the following form:

$$
E\left[e^{-\delta T-s\left(\sum_{i=1}^{N(t)} Y_{i}-c T\right)}\right]=\int_{-\infty}^{+\infty} e^{-s y} g_{\delta}(y) d y
$$

where $g_{\delta}(y)$ is the discounted density function of the increment $\sum_{i=1}^{N(t)} Y_{i}-c T$ between successive observation times. According to the variable $y$ being positive or negative, we can decompose $g_{\delta}(y)$ as follows:

$$
g_{\delta}(y)=g_{\delta,-}(-y) I_{\{y<0\}}+g_{\delta,+}(y) I_{\{y>0\}}, \quad-\infty<y<+\infty .
$$


Albrecher et al. [42] prove that as long as the density function of the claim amount satisfies $\tilde{f}_{Y}(s)=\frac{Q_{2, r-1}(s)}{Q_{1, r}(s)}, g_{\delta,-}(y)$ and $g_{\delta,+}(y)$ in the above formula have the following expressions:

$$
g_{\delta,-}(y)=\sum_{j=1}^{n} B_{j}^{*} \frac{y^{j-1} e^{-\rho_{\gamma} y}}{(j-1) !}, \quad g_{\delta,+}(y)=\sum_{i=1}^{r} \sum_{j=1}^{n} B_{i j} \frac{y^{j-1} e^{-k_{i} y}}{(j-1) !}
$$

where $\rho_{\gamma}$ is the only positive root of the equation $c s-(\lambda+\gamma+\delta)+\lambda \tilde{f}_{Y}(s)=0,\left\{-k_{i}\right\}_{i=1}^{r}$ is the negative root of the equation, and

$$
\begin{aligned}
B_{j}^{*} & =\left.(-1)^{n-j}\left(\frac{\gamma}{c}\right)^{n} \frac{1}{(n-j) !} \frac{d^{n-j}}{d s^{n-j}} \frac{\left[Q_{1, r}(s)\right]^{n}}{\prod_{l=1}^{r}\left(s+k_{l}\right)^{n}}\right|_{s=\rho_{\gamma}}, j=1,2, \ldots, n ; \\
B_{i j} & =\left.\left(\frac{\gamma}{c}\right)^{n} \frac{1}{(n-j) !} \frac{d^{n-j}}{d s^{n-j}} \frac{\left[Q_{1, r}(s)\right]^{n}}{\left(\rho_{\gamma}-s\right)^{n} \prod_{l=1, l \neq i}^{r}\left(s+k_{l}\right)^{n}}\right|_{s=-k_{i}}, \\
i & =1,2, \ldots, r ; j=1,2, \ldots, n .
\end{aligned}
$$

The layout of the paper is as follows: Sect. 2 presents the explicit expressions for the Gerber-Shiu function. Similarly, the expected discounted capital injection and the expected discounted dividend payments are studied in Sects. 3 and 4, respectively. In Sect. 5 we present some examples to show the effect of relevant parameters on the actuarial function.

\section{Gerber-Shiu function}

According to the first observation whether ruin occurs, the Gerber-Shiu function of the risk model with capital injection and barrier dividend strategy can be written as follows:

$$
\begin{aligned}
\Phi_{\delta}(u)= & \int_{0}^{\infty}\left[\Phi_{\delta}(u+y) I_{\left\{u+y \leq b_{2}\right\}}+\Phi_{\delta}\left(b_{2}\right) I_{\left\{u+y>b_{2}\right\}}\right] g_{\delta,-}(y) d y \\
& +\int_{0}^{u}\left[\Phi_{\delta}(u-y) I_{\left\{u-y \geq b_{1}\right\}}+\Phi_{\delta}\left(b_{1}\right) I_{\left\{u-y<b_{1}\right\}}\right] g_{\delta,+}(y) d y \\
& +\int_{u}^{\infty} w(y-u) g_{\delta,+}(y) d y .
\end{aligned}
$$

Taking the expression of $g_{\delta,-}(y)$ into the first integral of Eq. (15), we have

$$
\begin{aligned}
\int_{0}^{\infty} & {\left[\Phi_{\delta}(u+y) I_{\left\{u+y \leq b_{2}\right\}}+\Phi_{\delta}\left(b_{2}\right) I_{\left\{u+y>b_{2}\right\}}\right] g_{\delta,-}(y) d y } \\
= & \int_{0}^{b_{2}-u} \Phi_{\delta}(u+y) g_{\delta,-}(y) d y+\int_{b_{2}-u}^{\infty} \Phi_{\delta}\left(b_{2}\right) g_{\delta,-}(y) d y \\
= & \int_{0}^{b_{2}-u} \Phi_{\delta}(u+y) \sum_{j=1}^{n} B_{j}^{*} \frac{y^{j-1} e^{-\rho_{\gamma} y}}{(j-1) !} d y \\
& +\int_{b_{2}-u}^{\infty} \Phi_{\delta}\left(b_{2}\right) \sum_{j=1}^{n} B_{j}^{*} \frac{y^{j-1} e^{-\rho_{\gamma} y}}{(j-1) !} d y .
\end{aligned}
$$


For the expression $\int_{0}^{b_{2}-u} \Phi_{\delta}(u+y) \sum_{j=1}^{n} B_{j}^{*} \frac{j^{j-1} e^{-\rho_{\gamma} y}}{(j-1) !} d y$, let $z=u+y$. Then $y=z-u$, and thus we get

$$
\begin{aligned}
& \int_{0}^{b_{2}-u} \Phi_{\delta}(u+y) \sum_{j=1}^{n} B_{j}^{*} \frac{y^{j-1} e^{-\rho_{\gamma} y}}{(j-1) !} d y \\
& =\sum_{j=1}^{n} B_{j}^{*} \int_{0}^{b_{2}-u} \Phi_{\delta}(u+y) \frac{y^{j-1}}{(j-1) !} e^{-\rho_{\gamma} y} d y \\
& =\sum_{j=1}^{n} B_{j}^{*} \int_{u}^{b_{2}} \Phi_{\delta}(z) \frac{(z-u)^{j-1}}{(j-1) !} e^{-\rho_{\gamma}(z-u)} d z .
\end{aligned}
$$

For $\int_{b_{2}-u}^{\infty} \Phi_{\delta}\left(b_{2}\right) \sum_{j=1}^{n} B_{j}^{*} \frac{y^{j-1} e^{-\rho_{\gamma} y}}{(j-1) !} d y$, we have

$$
\begin{aligned}
& \Phi_{\delta}\left(b_{2}\right) \int_{b_{2}-u}^{\infty} \sum_{j=1}^{n} B_{j}^{*} \frac{y^{j-1} e^{-\rho_{\gamma} y}}{(j-1) !} d y \\
& =\Phi_{\delta}\left(b_{2}\right) \sum_{j=1}^{n} B_{j}^{*} \int_{b_{2}-u}^{\infty} \frac{y^{j-1} e^{-\rho_{\gamma} y}}{(j-1) !} d y \\
& =\Phi_{\delta}\left(b_{2}\right) \sum_{j=1}^{n} B_{j}^{*} \sum_{l=1}^{j} \frac{1}{\rho_{\gamma}^{j+1-l}} \frac{\left(b_{2}-u\right)^{l-1}}{(l-1) !} e^{-\rho_{\gamma}\left(b_{2}-u\right)} \\
& =\Phi_{\delta}\left(b_{2}\right) \sum_{j=1}^{n} B_{j}^{*} \sum_{l=1}^{j} \frac{1}{\rho_{\gamma}^{j+1-l}} \sum_{m=1}^{l} \frac{b_{2}^{l-m}}{(l-m) !} \frac{(-u)^{m-1}}{(m-1) !} e^{-\rho_{\gamma} b_{2}} e^{\rho_{\gamma} u} \\
& =\Phi_{\delta}\left(b_{2}\right) \sum_{m=1}^{n} \sum_{l=m}^{n} \sum_{j=l}^{n} B_{j}^{*} \frac{1}{\rho_{\gamma}^{j+1-l}} \frac{b_{2}^{l-m} e^{-\rho_{\gamma} b_{2}}}{(l-m) !} \frac{(-1)^{m-1} u^{m-1}}{(m-1) !} e^{\rho_{\gamma} u} .
\end{aligned}
$$

Substituting the expression of $g_{\delta,+}(y)$ into the second integral of Eq. (15), we have

$$
\begin{aligned}
& \int_{0}^{u}\left[\Phi_{\delta}(u-y) I_{\left\{u-y \geq b_{1}\right\}}+\Phi_{\delta}\left(b_{1}\right) I_{\left\{u-y<b_{1}\right\}}\right] g_{\delta,+}(y) d y \\
& \quad=\int_{0}^{u-b_{1}} \Phi_{\delta}(u-y) g_{\delta,+}(y) d y+\Phi_{\delta}\left(b_{1}\right) \int_{u-b_{1}}^{u} g_{\delta,+}(y) d y .
\end{aligned}
$$

For the expression $\int_{0}^{u-b_{1}} \Phi_{\delta}(u-y) g_{\delta,+}(y) d y$, let $z=u-y$. Then $y=u-z$, and thus

$$
\int_{0}^{u-b_{1}} \Phi_{\delta}(u-y) g_{\delta,+}(y) d y=\sum_{i=1}^{r} \sum_{j=1}^{n} B_{i j} \int_{b_{1}}^{u} \Phi_{\delta}(z) \frac{(u-z)^{j-1}}{(j-1) !} e^{-k_{i}(u-z)} d z .
$$

For $\Phi_{\delta}\left(b_{1}\right) \int_{u-b_{1}}^{u} g_{\delta,+}(y) d y$, we have

$$
\begin{aligned}
& \Phi_{\delta}\left(b_{1}\right) \int_{u-b_{1}}^{u} g_{\delta,+}(y) d y \\
& \quad=\Phi_{\delta}\left(b_{1}\right)\left[\int_{0}^{u} g_{\delta,+}(y) d y-\int_{0}^{u-b_{1}} g_{\delta,+}(y) d y\right]
\end{aligned}
$$




$$
\begin{aligned}
= & \Phi_{\delta}\left(b_{1}\right)\left\{\sum_{i=1}^{r} \sum_{j=1}^{n} B_{i j}\left[\frac{1}{k_{i}^{j}}-\sum_{l=1}^{j} \frac{1}{k_{i}^{j+1-l}} \frac{u^{l-1}}{(l-1) !} e^{-k_{i} u}\right]\right. \\
& \left.-\sum_{i=1}^{r} \sum_{j=1}^{n} B_{i j}\left[\frac{1}{k_{i}^{j}}-\sum_{l=1}^{j} \frac{1}{k_{i}^{j+1-l}} \frac{\left(u-b_{1}\right)^{l-1}}{(l-1) !} e^{-k_{i}\left(u-b_{1}\right)}\right]\right\} \\
= & \Phi_{\delta}\left(b_{1}\right) \sum_{i=1}^{r} \sum_{j=1}^{n} B_{i j} \sum_{l=1}^{j} \frac{1}{k_{i}^{j+1-l}}\left[\frac{\left(u-b_{1}\right)^{l-1}}{(l-1) !} e^{-k_{i}\left(u-b_{1}\right)}-\frac{u^{l-1}}{(l-1) !} e^{-k_{i} u}\right] \\
= & \Phi_{\delta}\left(b_{1}\right) \sum_{i=1}^{r} \sum_{j=1}^{n} B_{i j} \sum_{l=1}^{j} \frac{1}{k_{i}^{j+1-l}} \sum_{m=1}^{l} \frac{\left(-b_{1}\right)^{l-m}}{(l-m) !} \frac{u^{m-1}}{(m-1) !} e^{-k_{i} u} e^{k_{i} b_{1}} \\
& -\Phi_{\delta}\left(b_{1}\right) \sum_{i=1}^{r} \sum_{j=1}^{n} B_{i j} \sum_{l=1}^{j} \frac{1}{k_{i}^{j+1-l}} \frac{u^{l-1}}{(l-1) !} e^{-k_{i} u} \\
= & \Phi_{\delta}\left(b_{1}\right) \sum_{i=1}^{r} \sum_{m=1}^{n} \sum_{i=m}^{n} \sum_{j=l}^{n} B_{i j} \frac{1}{k_{i}^{j+1-l}} \frac{\left(-b_{l}\right)^{l-m}}{(l-m) !} e^{k_{i} b_{1}} \frac{u^{m-1}}{(m-1) !} e^{-k_{i} u} \\
& -\Phi_{\delta}\left(b_{1}\right) \sum_{i=1}^{r} \sum_{m=1}^{n} \sum_{j=m}^{n} B_{i j} \frac{1}{k_{i}^{j+1-m}} \frac{u^{m-1}}{(m-1) !} e^{-k_{i} u} .
\end{aligned}
$$

Now we consider the third integral of Eq. (15). Let $z=y-u$, then $y=z+u$, and thus the integral can be written as follows:

$$
\begin{aligned}
& \int_{u}^{\infty} w(y-u) g_{\delta,+}(y) d y \\
& \quad=\int_{0}^{\infty} w(z) g_{\delta,+}(u+z) d z \\
& \quad=\int_{0}^{\infty} w(z) \sum_{i=1}^{r} \sum_{j=1}^{n} B_{i j} \frac{(u+z)^{j-1}}{(j-1) !} e^{-k_{i}(u+z)} d z \\
& =\sum_{i=1}^{r} \sum_{j=1}^{n} B_{i j} \int_{0}^{\infty} w(z) \sum_{m=1}^{j} \frac{z^{j-m}}{(j-m) !} \frac{u^{m-1}}{(m-1) !} e^{-k_{i}(u+z)} d z \\
& =\sum_{i=1}^{r} \sum_{m=1}^{n} \sum_{j=m}^{n} B_{i j} \frac{u^{m-1}}{(m-1) !} e^{-k_{i} u} \int_{0}^{\infty} w(z) \frac{z^{j-m}}{(j-m) !} e^{-k_{i} z} d z .
\end{aligned}
$$

Applying the operator $\left(\frac{d}{d u}-\rho_{\gamma}\right)^{n} \prod_{i=1}^{r}\left(\frac{d}{d u}+k_{i}\right)^{n}$ simultaneously on both sides of Eq. (15), the left-hand side is clearly zero. The corresponding right-hand side result depends on the situation after the action of operators on the three right-hand side integrals. Due to $\left(\frac{d}{d u}-\rho_{\gamma}\right)^{m}\left(u^{m-1} e^{\rho_{\gamma} x}\right)=0$ and $\left(\frac{d}{d u}+k_{i}\right)^{l}\left(u^{l-1} e^{-k_{i} x}\right)=0$, the results of the above operators acting on (18), (21), and (22) are 0 , and then we have

$$
\begin{aligned}
& \left(\frac{d}{d u}-\rho_{\gamma}\right)^{j} \int_{u}^{b_{2}} \Phi_{\delta}(z)(-1)^{j-1} \frac{(u-z)^{j-1}}{(j-1) !} e^{\rho_{\gamma}(u-z)} d z=(-1)^{j} \Phi_{\delta}(u), \\
& \left(\frac{d}{d u}+k_{i}\right)^{j} \int_{b_{1}}^{u} \Phi_{\delta}(z) \frac{(u-z)^{j-1}}{(j-1) !} e^{-k_{i}(u-z)} d z=\Phi_{\delta}(u) .
\end{aligned}
$$


Therefore, the higher-order differential equation of $\Phi_{\delta}(u)$ can be obtained as

$$
\begin{aligned}
\left(\frac{d}{d u}-\rho_{\gamma}\right)^{n} \prod_{i=1}^{r}\left(\frac{d}{d u}+k_{i}\right)^{n} \Phi_{\delta}(u) & \\
= & \prod_{i=1}^{r}\left(\frac{d}{d u}+k_{i}\right)^{n}\left(\frac{d}{d u}-\rho_{\gamma}\right)^{n-j}\left(\frac{d}{d u}-\rho_{\gamma}\right)^{j} \sum_{j=1}^{n} B_{j}^{*} \int_{u}^{b_{2}} \Phi_{\delta}(z) \frac{(z-u)^{j-1}}{(j-1) !} e^{-\rho_{\gamma}(z-u)} d z \\
& +\left(\frac{d}{d u}-\rho_{\gamma}\right)^{n} \prod_{i=1}^{r}\left(\frac{d}{d u}+k_{i}\right)^{n-j}\left(\frac{d}{d u}+k_{i}\right)^{j} \\
& \times \sum_{i=1}^{r} \sum_{j=1}^{n} B_{i j} \int_{b_{1}}^{u} \Phi_{\delta}(z) \frac{(u-z)^{j-1}}{(j-1) !} e^{-k_{i}(u-z)} d z \\
= & \sum_{j=1}^{n}(-1)^{j} B_{j}^{*} \prod_{i=1}^{r}\left(\frac{d}{d u}+k_{i}\right)^{n}\left(\frac{d}{d u}-\rho_{\gamma}\right)^{n-j} \Phi_{\delta}(u) \\
& +\sum_{i=1}^{r} \sum_{j=1}^{n} B_{i j}\left(\frac{d}{d u}-\rho_{\gamma}\right)^{n} \prod_{i=1}^{r}\left(\frac{d}{d u}+k_{i}\right)^{n-j} \Phi_{\delta}(u)
\end{aligned}
$$

Solving the above equation, the general solution form of $\Phi_{\delta}(u)$ can be obtained as follows:

$$
\Phi_{\delta}(u)=\sum_{z=1}^{n(r+1)} C_{z} e^{\alpha_{z} u}
$$

where $\alpha_{Z}$ is the characteristic root corresponding to the above higher-order differential equation, and Albrecher et al. [42] proved that $\alpha_{Z}$ is the root of the equation $E\left[e^{-\delta T-s\left(\sum_{i=1}^{N(t)} Y_{i}-c T\right)}\right]=1$ with respect to $s$. We now substitute formula (24) into Eq. (15), and calculate the three integrals, which are recorded as $H_{1}, H_{2}, H_{3}$, respectively. The first integral is calculated as follows:

$$
\begin{aligned}
H_{1} & =\int_{0}^{\infty}\left[\Phi_{\delta}(u+y) I_{\left\{u+y \leq b_{2}\right\}}+\Phi_{\delta}\left(b_{2}\right) I_{\left\{u+y>b_{2}\right\}}\right] g_{\delta,-}(y) d y \\
& =\int_{0}^{b_{2}-u} \Phi_{\delta}(u+y) g_{\delta,-}(y) d y+\Phi_{\delta}\left(b_{2}\right) \int_{b_{2}-u}^{\infty} g_{\delta,-}(y) d y,
\end{aligned}
$$

where

$$
\begin{aligned}
& \int_{0}^{b_{2}-u} \Phi_{\delta}(u+y) g_{\delta,-}(y) d y \\
& \quad=\int_{0}^{b_{2}-u} \sum_{z=1}^{n(r+1)} C_{z} e^{\alpha_{z}(u+y)} \sum_{j=1}^{n} B_{j}^{*} \frac{y^{j-1} e^{-\rho_{\gamma} y}}{(j-1) !} d y \\
& =\sum_{z=1}^{n(r+1)} C_{z} e^{\alpha_{z} u} \sum_{j=1}^{n} B_{j}^{*} \int_{0}^{b_{2}-u} \frac{y^{j-1}}{(j-1) !} e^{-\left(\rho_{\gamma}-\alpha_{z}\right) y} d y \\
& =\sum_{z=1}^{n(r+1)} C_{z} e^{\alpha_{z} u} \sum_{j=1}^{n} B_{j}^{*}\left[\frac{1}{\left(\rho_{\gamma}-\alpha_{z}\right)^{j}}-\sum_{i=1}^{j} \frac{1}{\left(\rho_{\gamma}-\alpha_{z}\right)^{j+1-l}} \frac{\left(b_{2}-u\right)^{l-1}}{(l-1) !} e^{-\left(\rho_{\gamma}-\alpha_{z}\right)\left(b_{2}-u\right)}\right]
\end{aligned}
$$




$$
\begin{aligned}
= & \sum_{z=1}^{n(r+1)} C_{z} e^{\alpha_{z} u} \sum_{j=1}^{n} B_{j}^{*} \frac{1}{\left(\rho_{\gamma}-\alpha_{z}\right)^{j}}-\sum_{z=1}^{n(r+1)} C_{z} e^{\alpha_{z} u} \sum_{j=1}^{n} B_{j}^{*} \sum_{l=1}^{j} \frac{\left(b_{2}-u\right)^{l-1} e^{-\left(\rho_{\gamma}-\alpha_{z}\right)\left(b_{2}-u\right)}}{\left(\rho_{\gamma}-\alpha_{z}\right)^{j+1-l}(l-1) !} \\
= & \sum_{z=1}^{n(r+1)} C_{z} e^{\alpha_{z} u} \sum_{j=1}^{n} B_{j}^{*} \frac{1}{\left(\rho_{\gamma}-\alpha_{z}\right)^{j}}-\sum_{z=1}^{n(r+1)} C_{z} \sum_{l=1}^{n} \sum_{j=l}^{n} B_{j}^{*} \frac{\left(b_{2}-u\right)^{l-1} e^{-\left(\rho_{\gamma}-\alpha_{z}\right)\left(b_{2}-u\right)}}{\left(\rho_{\gamma}-\alpha_{z}\right)^{j+1-l}(l-1) !} \\
= & \sum_{z=1}^{n(r+1)} C_{z} e^{\alpha_{z} u} \sum_{j=1}^{n} B_{j}^{*} \frac{1}{\left(\rho_{\gamma}-\alpha_{z}\right)^{j}} \\
& -\sum_{z=1}^{n(r+1)} C_{z} \sum_{m=1}^{n} \sum_{l=m}^{n} \sum_{j=l}^{n} B_{j}^{*} \frac{1}{\left(\rho_{\gamma}-\alpha_{z}\right)^{j+1-l}} \frac{b_{2}^{l-m}}{(l-m) !} e^{\left(\alpha_{z}-\rho_{\gamma}\right) b_{2}} \frac{(-1)^{m-1} u^{m-1}}{(m-1) !} e^{\rho_{\gamma} u},
\end{aligned}
$$

and

$$
\begin{aligned}
& \Phi_{\delta}\left(b_{2}\right) \int_{b_{2}-u}^{\infty} g_{\delta,-}(y) d y \\
& =\Phi_{\delta}\left(b_{2}\right) \sum_{m=1}^{n} \sum_{l=m}^{n} \sum_{j=l}^{n} B_{j}^{*} \frac{1}{\rho_{\gamma}^{j+1-l}} \frac{b_{2}^{l-m}}{(l-m) !} e^{-\rho_{\gamma} b_{2}}(-1)^{m-1} \frac{u^{m-1}}{(m-1) !} e^{\rho_{\gamma} u} \\
& =\sum_{z=1}^{n(r+1)} C_{z} \sum_{m=1}^{n} \sum_{l=m}^{n} \sum_{j=l}^{n} B_{j}^{*} \frac{1}{\rho_{\gamma}^{j+1-l}} \frac{b_{2}^{l-m}}{(l-m) !} e^{\left(\alpha_{z}-\rho_{\gamma}\right) b_{2}}(-1)^{m-1} \frac{u^{m-1}}{(m-1) !} e^{\rho_{\gamma} u} .
\end{aligned}
$$

Thus, we have

$$
\begin{aligned}
H_{1}= & \int_{0}^{\infty}\left[\Phi_{\delta}(u+y) I_{\left\{u+y \leq b_{2}\right\}}+\Phi_{\delta}\left(b_{2}\right) I_{\left\{u+y>b_{2}\right\}}\right] g_{\delta,-}(y) d y \\
= & \sum_{z=1}^{n(r+1)} C_{z} e^{\alpha_{z} u} \sum_{j=1}^{n} B_{j}^{*} \frac{1}{\left(\rho_{\gamma}-\alpha_{z}\right)^{j}} \\
& +\sum_{z=1}^{n(r+1)} C_{z} \sum_{m=1}^{n} \sum_{l=m}^{n} \sum_{j=l}^{n} B_{j}^{*}\left(\frac{1}{\rho_{\gamma}^{j+1-l}}-\frac{1}{\left(\rho_{\gamma}-\alpha_{z}\right)^{j+1-l}}\right) \\
& \times \frac{b_{2}^{l-m} e^{\left(\alpha_{z}-\rho_{\gamma}\right) b_{2}}}{(l-m) !} \frac{(-1)^{m-1} u^{m-1}}{(m-1) !} e^{\rho_{\gamma} u}
\end{aligned}
$$

The second integral is calculated as follows:

$$
\begin{aligned}
H_{2} & =\int_{0}^{u}\left[\Phi_{\delta}(u-y) I_{\left\{u-y \geq b_{1}\right\}}+\Phi_{\delta}\left(b_{1}\right) I_{\left\{u-y<b_{1}\right\}}\right] g_{\delta,+}(y) d y \\
& =\int_{0}^{u-b_{1}} \Phi_{\delta}(u-y) g_{\delta,+}(y) d y+\Phi_{\delta}\left(b_{1}\right) \int_{u-b_{1}}^{u} g_{\delta,+}(y) d y,
\end{aligned}
$$

where

$$
\begin{aligned}
& \int_{0}^{u-b_{1}} \Phi_{\delta}(u-y) g_{\delta,+}(y) d y \\
& \quad=\int_{0}^{u-b_{1}} \sum_{z=1}^{n(r+1)} C_{z} e^{\alpha_{z}(u-y)} \sum_{i=1}^{r} \sum_{j=1}^{n} B_{i j} \frac{y^{j-1}}{(j-1) !} e^{-k_{i} y} d y
\end{aligned}
$$




$$
\begin{aligned}
& =\sum_{z=1}^{n(r+1)} C_{z} e^{\alpha_{z} u} \sum_{i=1}^{r} \sum_{j=1}^{n} B_{i j} \int_{0}^{u-b_{1}} \frac{y^{j-1}}{(j-1) !} e^{-\left(k_{i}+\alpha_{z}\right) y} d y \\
& =\sum_{z=1}^{n(r+1)} C_{z} e^{\alpha_{z} u} \sum_{i=1}^{r} \sum_{j=1}^{n} B_{i j}\left[\frac{1}{\left(k_{i}+\alpha_{z}\right)^{j}}-\sum_{l=1}^{j} \frac{1}{\left(k_{i}+\alpha_{z}\right)^{j+1-l}} \frac{\left(u-b_{1}\right)^{l-1}}{(l-1) !} e^{-\left(k_{i}+\alpha_{z}\right)\left(u-b_{1}\right)}\right] \\
& =\sum_{z=1}^{n(r+1)} C_{z} e^{\alpha_{z} u} \sum_{i=1}^{r} \sum_{j=1}^{n} B_{i j} \frac{1}{\left(k_{i}+\alpha_{z}\right)^{j}} \\
& -\sum_{z=1}^{n(r+1)} C_{z} e^{\alpha_{z} u} \sum_{i=1}^{r} \sum_{j=1}^{n} B_{i j} \sum_{l=1}^{j} \frac{1}{\left(k_{i}+\alpha_{z}\right)^{j+1-l}} \frac{\left(u-b_{1}\right)^{l-1}}{(l-1) !} e^{-\left(k_{i}+\alpha_{z}\right) u} e^{\left(k_{i}+\alpha_{z}\right) b_{1}} \\
& =\sum_{z=1}^{n(r+1)} C_{z} e^{\alpha_{z} u} \sum_{i=1}^{r} \sum_{j=1}^{n} B_{i j} \frac{1}{\left(k_{i}+\alpha_{z}\right)^{j}} \\
& -\sum_{z=1}^{n(r+1)} C_{z} \sum_{i=1}^{r} \sum_{l=1}^{n} \sum_{j=l}^{n} B_{i j} \frac{1}{\left(k_{i}+\alpha_{z}\right)^{j+1-l}} \frac{\left(u-b_{1}\right)^{l-1}}{(l-1) !} e^{-k_{i} u} e^{\left(k_{i}+\alpha_{z}\right) b_{1}} \\
& =\sum_{z=1}^{n(r+1)} C_{z} e^{\alpha_{z} u} \sum_{i=1}^{r} \sum_{j=1}^{n} B_{i j} \frac{1}{\left(k_{i}+\alpha_{z}\right)^{j}} \\
& -\sum_{z=1}^{n(r+1)} C_{z} \sum_{i=1}^{r} \sum_{m=1}^{n} \sum_{l=m}^{n} \sum_{j=l}^{n} B_{i j} \frac{1}{\left(k_{i}+\alpha_{z}\right)^{j+1-l}} \frac{\left(-b_{1}\right)^{l-m}}{(l-m) !} e^{\left(k_{i}+\alpha_{z}\right) b_{1}} \frac{u^{m-1}}{(m-1) !} e^{-k_{i} u}
\end{aligned}
$$

and

$$
\begin{aligned}
& \Phi_{\delta}\left(b_{1}\right) \int_{u-b_{1}}^{u} g_{\delta,+}(y) d y \\
&=\Phi_{\delta}\left(b_{1}\right) \sum_{i=1}^{r} \sum_{m=1}^{n} \sum_{l=m}^{n} \sum_{j=l}^{n} B_{i j} \frac{1}{k_{i}^{j+1-l}} \frac{\left(-b_{1}\right)^{l-m}}{(l-m) !} e^{k_{i} b_{1}} \frac{u^{m-1}}{(m-1) !} e^{-k_{i} u} \\
& \quad-\Phi_{\delta}\left(b_{1}\right) \sum_{i=1}^{r} \sum_{m=1}^{n} \sum_{j=m}^{n} B_{i j} \frac{1}{k_{i}^{j+1-m}} \frac{u^{m-1}}{(m-1) !} e^{-k_{i} u} \\
&=\Phi_{\delta}\left(b_{1}\right) \sum_{i=1}^{r} \sum_{m=1}^{n} \sum_{l=m}^{n} \sum_{j=l}^{n} B_{i j} \frac{1}{k_{i}^{j+1-l}} \frac{\left(-b_{1}\right)}{(l-m) !} e^{k_{i} b_{1}} \frac{u^{m-1}}{(m-1) !} e^{-k_{i} u} \\
&-\Phi_{\delta}\left(b_{1}\right) \sum_{i=1}^{r} \sum_{m=1}^{n} \sum_{j=m}^{n} B_{i j} \frac{1}{k_{i}^{j+1-m}} \frac{u^{m-1}}{(m-1) !} e^{-k_{i} u} \\
& n(r+1) \\
&= \sum_{z=1}^{r} C_{z} \sum_{i=1}^{r} \sum_{m=1}^{n}\left[\sum_{l=m}^{n} \sum_{j=l}^{n} B_{i j} \frac{1}{k_{i}^{j+1-l}} e^{\left(k_{i}+\alpha_{z}\right) b_{1}} \frac{\left(-b_{1}\right)^{l-m}}{(l-m) !}-\sum_{j=m}^{n} B_{i j} \frac{e^{\alpha_{z} b_{1}}}{k_{i}^{j+1-m}}\right] \\
& \times \frac{u^{m-1}}{(m-1) !} e^{-k_{i} u} .
\end{aligned}
$$


Thus, we have

$$
\begin{aligned}
& H_{2}=\int_{0}^{u}\left[\Phi_{\delta}(u-y) I_{\left\{u-y \geq b_{1}\right\}}+\Phi_{\delta}\left(b_{1}\right) I_{\left\{u-y<b_{1}\right\}}\right] g_{\delta,+}(y) d y \\
& =\sum_{z=1}^{n(r+1)} C_{z} e^{\alpha_{z} u} \sum_{i=1}^{r} \sum_{j=1}^{n} B_{i j} \frac{1}{\left(k_{i}+\alpha_{z}\right)^{j}} \\
& -\sum_{z=1}^{n(r+1)} C_{z} \sum_{i=1}^{r} \sum_{m=1}^{n} \sum_{l=m}^{n} \sum_{j=l}^{n} B_{i j} \frac{1}{\left(k_{i}+\alpha_{z}\right)^{j+1-l}} \frac{\left(-b_{1}\right)^{l-m}}{(l-m) !} e^{\left(k_{i}+\alpha_{z}\right) b_{1}} \frac{u^{m-1}}{(m-1) !} e^{-k_{i} u} \\
& +\sum_{z=1}^{n(r+1)} C_{z} \sum_{i=1}^{r} \sum_{m=1}^{n}\left[\sum_{l=m}^{n} \sum_{j=l}^{n} B_{i j} \frac{e^{\left(k_{i}+\alpha_{z}\right) b_{1}}}{k_{i}^{j+1-l}} \frac{\left(-b_{1}\right)^{l-m}}{(l-m) !}-\sum_{j=m}^{n} B_{i j} \frac{1}{k_{i}^{j+1-m}} e^{\alpha_{z} b_{1}}\right] \\
& \times \frac{u^{m-1}}{(m-1) !} e^{-k_{i} u} \\
& =\sum_{z=1}^{n(r+1)} C_{z} e^{\alpha_{z} x} \sum_{i=1}^{r} \sum_{j=1}^{n} B_{i j} \frac{1}{\left(k_{i}+\alpha_{z}\right)^{j}} \\
& +\sum_{z=1}^{n(r+1)} C_{z} \sum_{i=1}^{r} \sum_{m=1}^{n}\left[\sum_{l=m}^{n} \sum_{j=l}^{n} B_{i j}\left(\frac{1}{k_{i}^{j+1-l}}-\frac{1}{\left(k_{i}+\alpha_{z}\right)^{j+1-l}}\right) e^{\left(k_{i}+\alpha_{z}\right) b_{1}} \frac{\left(-b_{1}\right)^{l-m}}{(l-m) !}\right. \\
& \left.-\sum_{j=m}^{n} B_{i j} \frac{1}{k_{i}^{j+1-l}} e^{\alpha_{z} b_{1}}\right] \frac{u^{m-1}}{(m-1) !} e^{-k_{i} u} \text {. }
\end{aligned}
$$

The third integral is calculated as follows:

$$
H_{3}=\int_{u}^{\infty} w(y-u) g_{\delta,+}(y) d y=\sum_{i=1}^{r} \sum_{j=1}^{n} B_{i j} \int_{u}^{\infty} w(y-u) \frac{y^{j-1} e^{-k_{i} y}}{(j-1) !} d y
$$

Plugging the integrals (25)-(27) into the Eq. (15), we have

$$
\begin{aligned}
\Phi_{\delta}(u)= & \sum_{z=1}^{n(r+1)} C_{z} e^{\alpha_{z} u}\left[\sum_{j=1}^{n} B_{j}^{*} \frac{1}{\left(\rho_{\gamma}-\alpha_{z}\right)^{j}}+\sum_{i=1}^{r} \sum_{j=1}^{n} B_{i j} \frac{1}{\left(k_{i}+\alpha_{z}\right)^{j}}\right] \\
& +\sum_{m=1}^{n} \sum_{z=1}^{n(r+1)} C_{z} \sum_{l=m}^{n} \sum_{j=l}^{n} B_{j}^{*}\left(\frac{1}{\rho_{\gamma}^{j+1-l}}-\frac{1}{\left(\rho_{\gamma}-\alpha_{z}\right)^{j+1-l}}\right) \\
& +\frac{b_{2}^{l-m} e^{\left(\alpha_{z}-\rho_{\gamma}\right) b_{2}}}{(l-1)^{m-1} u^{m-1}} \frac{(m-1) !}{\left(m-\rho_{\gamma} u\right.} \\
& +\sum_{i=1}^{r} \sum_{m=1}^{n}\left[\sum_{z=1}^{n(r+1)} C_{z} \sum_{l=m}^{n} \sum_{j=l}^{n} B_{i j}\left(\frac{1}{k_{i}^{j+1-l}}-\frac{1}{\left(k_{i}+\alpha_{z}\right)^{j+1-l}}\right) e^{\left(k_{i}+\alpha_{z}\right) b_{1}} \frac{\left(-b_{1}\right)^{l-m}}{(l-m) !}\right. \\
& \left.-\sum_{z=1}^{n(r+1)} C_{z} \sum_{j=m}^{n} B_{i j} \frac{1}{k_{i}^{j+1-l}} e^{\alpha_{z} b_{1}}\right] \frac{u^{m-1}}{(m-1) !} e^{-k_{i} u} \\
& +\sum_{i=1}^{r} \sum_{j=1}^{n} B_{i j} \int_{u}^{\infty} w(y-u) \frac{y^{j-1} e^{-k_{i} y}}{(j-1) !} d y .
\end{aligned}
$$


When the penalty function $w(y-u)$ is determined, $n(r+1)$ equations with coefficients $C_{z}$ can be obtained from Eq. (28). Based on this, the parameters contained in $\Phi_{\delta}(u)=$ $\sum_{z=1}^{n(r+1)} C_{z} e^{\alpha_{z} u}$ can be obtained, and then the corresponding Gerber-Shiu function can be obtained.

\section{Expected discounted capital injection}

Similar to solving for the Gerber-Shiu function, we can get the integral equation satisfied by the expected discounted capital injection

$$
\begin{aligned}
\Psi(u)= & \int_{0}^{\infty}\left[\Psi(u+y) I_{\left\{u+y \leq b_{2}\right\}}+\Psi\left(b_{2}\right) I_{\left\{u+y>b_{2}\right\}}\right] g_{\delta,-}(y) d y \\
& +\int_{0}^{x}\left[\Psi(u-y) I_{\left\{u-y \geq b_{1}\right\}}+\left(\chi_{1}\left(b_{1}-(u-y)\right)+\Psi\left(b_{1}\right)\right) I_{\left\{u-y<b_{1}\right\}}\right] \\
& \times g_{\delta,+}(y) d y .
\end{aligned}
$$

For the following integral

$$
\int_{0}^{u} \chi_{1}\left(b_{1}-(u-y)\right) I_{\left\{u-y<b_{1}\right\}} g_{\delta,+}(y) d y=\int_{u-b_{1}}^{u} \chi_{1}\left(b_{1}-(u-y)\right) g_{\delta,+}(y) d y,
$$

let $z=b_{1}-(u-y)$, and then $y=z-b_{1}+u$. After bringing $g_{\delta,+}(y)$ into integral (30), the above integral can be simplified as follows:

$$
\begin{aligned}
\int_{0}^{u} & \chi_{1}\left(b_{1}-(u-y)\right) I_{\left\{u-y<b_{1}\right.} g_{\delta,+}(y) d y \\
& =\int_{u-b_{1}}^{u} \chi_{1}\left(b_{1}-(u-y)\right) g_{\delta,+}(y) d y \\
& =\int_{0}^{b_{1}} \chi_{1}(z) g_{\delta,+}\left(z-b_{1}+u\right) d z \\
& =\sum_{i=1}^{r} \sum_{j=1}^{n} B_{i j} e^{-k_{i}\left(u-b_{1}\right)} \int_{0}^{b_{1}} \chi_{1}(z) \frac{\left(z+\left(x-b_{1}\right)\right)^{j-1}}{(j-1) !} e^{-k_{i} z} d z \\
& =\sum_{i=1}^{r} \sum_{j=1}^{n} B_{i j} e^{-k_{i}\left(u-b_{1}\right)} \int_{0}^{b_{1}} \chi_{1}(z) \sum_{l=1}^{j} \frac{\left(x-b_{1}\right)^{l-1} z^{j-l}}{(l-1) !(j-l) !} e^{-k_{i} z} d z \\
& =\sum_{i=1}^{r} \sum_{j=1}^{n} B_{i j} e^{-k_{i}\left(u-b_{1}\right)} \sum_{l=1}^{j} \frac{\left(u-b_{1}\right)^{l-1}}{(l-1) !} \int_{0}^{b_{1}} \chi_{1}(z) \frac{z^{j-l}}{(j-l) !} e^{-k_{i} z} d z \\
& =\sum_{i=1}^{r} \sum_{j=1}^{n} B_{i j} e^{-k_{i}\left(u-b_{1}\right)} \sum_{l=1}^{j} \sum_{m=1}^{l} \frac{u^{m-1}\left(-b_{1}\right)^{l-m}}{(m-1) !(l-m) !} \int_{0}^{b_{1}} \chi_{1}(z) \frac{z^{j-l}}{(j-l) !} e^{-k_{i} z} d z \\
& =\sum_{i=1}^{r} \sum_{m=1}^{n} \sum_{l=m}^{n} \sum_{j=l}^{n} B_{i j} e^{k_{i} b_{1}} \frac{\left(-b_{1}\right)^{l-m}}{(l-m) !} \frac{u^{m-1}}{(m-1) !} e^{-k_{i} u} \int_{0}^{b_{1}} \chi_{1}(z) \frac{z^{j-l}}{(j-l) !} e^{-k_{i} z} d z .
\end{aligned}
$$

The operation with other integrals is exactly the same as that of the related integral in the Gerber-Shiu function. By applying the operator $\left(\frac{d}{d u}-\rho_{\gamma}\right)^{n} \prod_{i=1}^{r}\left(\frac{d}{d u}+k_{i}\right)^{n}$ on both sides of Eq. (29) at the same time, a higher-order differential equation for $\Psi(u)$ can be obtained. 
The general solution to this equation can be obtained as follows:

$$
\Psi(u)=\sum_{z=1}^{n(r+1)} A_{z} e^{\alpha_{z} u},
$$

where $\alpha_{Z}$ is also the characteristic root corresponding to the above higher-order differential equation. Now substitute formula (32) into Eq. (29), and calculate the two integrals on the right. The first integral in Eq. (29) can be directly obtained by using the result for the related integral in Eq. (15) as

$$
\begin{aligned}
\int_{0}^{\infty}\left[\Psi(u+y) I_{\left\{u+y \leq b_{2}\right\}}+\Psi\left(b_{2}\right) I_{\left\{u+y>b_{2}\right\}}\right] g_{\delta,-}(y) d y \\
=\int_{0}^{b_{2}-u} \Psi(u+y) g_{\delta,-}(y) d y+\Psi\left(b_{2}\right) \int_{b_{2}-u}^{\infty} g_{\delta,-}(y) d y \\
=\sum_{z=1}^{n(r+1)} A_{z} e^{\alpha_{z} u} \sum_{j=1}^{n} B_{j}^{*} \frac{1}{\left(\rho_{\gamma}-\alpha_{z}\right)^{j}} \\
\quad+\sum_{z=1}^{n(r+1)} A_{z} \sum_{m=1}^{n} \sum_{l=m}^{n} \sum_{j=l}^{n} B_{j}^{*}\left(\frac{1}{\rho_{\gamma}^{j+1-l}}-\frac{1}{\left(\rho_{\gamma}-\alpha_{z}\right)^{j+1-l}}\right) \\
\quad \times \frac{b_{2}^{l-m} e^{\left(\alpha_{z}-\rho_{\gamma}\right) b_{2}}}{(l-m) !} \frac{(-1)^{m-1} u^{m-1}}{(m-1) !} e^{\rho_{\gamma} u} .
\end{aligned}
$$

By (31), the second integral of Eq. (29) is calculated as follows:

$$
\begin{aligned}
\int_{0}^{u} & {\left[\Psi(u-y) I_{\left\{u-y \geq b_{1}\right\}}+\left[\chi_{1}\left(b_{1}-(u-y)\right)+\Psi\left(b_{1}\right)\right] I_{\left\{u-y<b_{1}\right\}}\right] g_{\delta,+}(y) d y } \\
= & \int_{0}^{u-b_{1}} g_{\delta,+}(y) \Psi(u-y) d y+\int_{u-b_{1}}^{u} g_{\delta,+}(y)\left[\chi_{1}\left(b_{1}-(u-y)\right)+\Psi\left(b_{1}\right)\right] d y \\
= & \sum_{z=1}^{n(r+1)} A_{z} e^{\alpha_{z} u} \sum_{i=1}^{r} \sum_{j=1}^{n} B_{i j} \frac{1}{\left(k_{i}+\alpha_{z}\right)^{j}} \\
& +\sum_{i=1}^{r} \sum_{m=1}^{n} \sum_{l=m}^{n} \sum_{j=l}^{n} B_{i j} e^{k_{i} b_{1}} \frac{\left(-b_{1}\right)^{l-m}}{(l-m) !} \frac{u^{m-1}}{(m-1) !} e^{-k_{i} u} \int_{0}^{b_{1}} \chi_{1}(z) \frac{z^{j-l}}{(j-l) !} e^{-k_{i} z} d z \\
& +\sum_{z=1}^{n(r+1)} A_{z} \sum_{i=1}^{r} \sum_{m=1}^{n}\left[\sum_{l=m}^{n} \sum_{j=l}^{n} B_{i j}\left(\frac{1}{k_{i}^{j+1-l}}-\frac{1}{\left(k_{i}+\alpha_{z}\right)^{j+1-l}}\right) e^{\left(k_{i}+\alpha_{z}\right) b_{1}} \frac{\left(-b_{1}\right)^{l-m}}{(l-m) !}\right. \\
& \left.\quad-\sum_{j=m}^{n} B_{i j} \frac{1}{k_{i}^{j+1-m}} e^{\alpha_{z} b_{1}}\right] \frac{u^{m-1}}{(m-1) !} e^{-k_{i} u} .
\end{aligned}
$$

Substituting the two integrals (33) and (34) into Eq. (29), we have

$$
\begin{aligned}
\Psi(u)= & \sum_{z=1}^{n(r+1)} A_{z} e^{\alpha_{z} u} \\
& =\sum_{z=1}^{n(r+1)} A_{z} e^{\alpha_{z} u} \sum_{j=1}^{n} B_{j}^{*} \frac{1}{\left(\rho_{\gamma}-\alpha_{z}\right)^{j}}
\end{aligned}
$$




$$
\begin{aligned}
& +\sum_{z=1}^{n(r+1)} A_{z} \sum_{m=1}^{n} \sum_{l=m}^{n} \sum_{j=l}^{n} B_{j}^{*}\left(\frac{1}{\rho_{\gamma}^{j+1-l}}-\frac{1}{\left(\rho_{\gamma}-\alpha_{z}\right)^{j+1-l}}\right) \\
& \times \frac{b_{2}^{l-m} e^{\left(\alpha_{z}-\rho_{\gamma}\right) b_{2}}}{(l-m) !} \frac{(-1)^{m-1} u^{m-1}}{(m-1) !} e^{\rho_{\gamma} u} \\
& +\sum_{z=1}^{n(r+1)} A_{z} e^{\alpha_{z} u} \sum_{i=1}^{r} \sum_{j=l}^{n} B_{i j} \frac{1}{\left(k_{i}+\alpha_{z}\right)^{j}} \\
& +\sum_{i=1}^{r} \sum_{m=1}^{n} \sum_{l=m}^{n} \sum_{j=l}^{n} B_{i j} e^{k_{i} b_{1}} \frac{\left(-b_{1}\right)^{l-m}}{(l-m) !} \frac{u^{m-1}}{(m-1) !} e^{-k_{i} u} \int_{0}^{b_{1}} \chi_{1}(z) \frac{z^{j-l}}{(j-l) !} e^{-k_{i} z} d z \\
& +\sum_{z=1}^{n(r+1)} A_{z} \sum_{i=1}^{r} \sum_{m=1}^{n}\left[\sum_{l=m}^{n} \sum_{j=l}^{n} B_{i j}\left(\frac{1}{k_{i}^{j+1-l}}-\frac{1}{\left(k_{i}+\alpha_{z}\right)^{j+1-l}}\right)\right. \\
& \times e^{\left(k_{i}+\alpha_{z}\right) b_{1}} \frac{\left(-b_{1}\right)^{l-m}}{(l-m) !} \\
& \left.-\sum_{j=m}^{n} B_{i j} \frac{1}{k_{i}^{j+1-m}} e^{\alpha_{z} b_{1}}\right] \frac{u^{m-1}}{(m-1) !} e^{-k_{i} u} \\
& =\sum_{z=1}^{n(r+1)} A_{z} e^{\alpha_{z} u}\left[\sum_{j=1}^{n} B_{j}^{*} \frac{1}{\left(\rho_{\gamma}-\alpha_{z}\right)^{j}}+\sum_{i=1}^{r} \sum_{j=l}^{n} B_{i j} \frac{1}{\left(k_{i}+\alpha_{z}\right)^{j}}\right] \\
& +\sum_{z=1}^{n(r+1)} A_{z} \sum_{m=1}^{n} \sum_{l=m}^{n} \sum_{j=l}^{n} B_{j}^{*}\left(\frac{1}{\rho_{\gamma}^{j+1-l}}-\frac{1}{\left(\rho_{\gamma}-\alpha_{z}\right)^{j+1-l}}\right) \\
& \times \frac{b_{2}^{l-m} e^{\left(\alpha_{z}-\rho_{\gamma}\right) b_{2}}}{(l-m) !} \frac{(-1)^{m-1} u^{m-1}}{(m-1) !} e^{\rho_{\gamma} u} \\
& +\sum_{z=1}^{n(r+1)} A_{z} \sum_{i=1}^{r} \sum_{m=1}^{n}\left[\sum_{l=m}^{n} \sum_{j=l}^{n} B_{i j}\left(\frac{1}{k_{i}^{j+1-l}}-\frac{1}{\left(k_{i}+\alpha_{z}\right)^{j+1-l}}\right)\right. \\
& \times e^{\left(k_{i}+\alpha_{z}\right) b_{1}} \frac{\left(-b_{1}\right)^{l-m}}{(l-m) !} \\
& \left.-\sum_{j=m}^{n} B_{i j} \frac{1}{k_{i}^{j+1-m}} e^{\alpha_{z} b_{1}}\right] \frac{u^{m-1}}{(m-1) !} e^{-k_{i} u} \\
& +\sum_{i=1}^{r} \sum_{m=1}^{n} \sum_{l=m}^{n} \sum_{j=l}^{n} B_{i j} e^{k_{i} b_{1}} \frac{\left(-b_{1}\right)^{l-m}}{(l-m) !} \frac{u^{m-1}}{(m-1) !} e^{-k_{i} u} \\
& \times \int_{0}^{b_{1}} \chi_{1}(z) \frac{z^{j-l}}{(j-l) !} e^{-k_{i} z} d z
\end{aligned}
$$

The result of the above integral will depend on the form of the loss function $\chi_{1}(x)$. When the form of function $\chi_{1}(x)$ is given, the specific result of the above integral can be calculated. It is consistent with the solution method for the Gerber-Shiu function. After combining similar terms, the equation satisfied by $n(r+1)$ coefficients can be obtained according to the above result. Therefore, all parameters contained in $\Psi(u)=\sum_{z=1}^{n(r+1)} A_{z} e^{\alpha_{z} u}$ can be found, and then the expression of $\Psi(u)$ can be obtained. 


\section{Expected discounted dividend payments}

Similarly as for the Gerber-Shiu function, we can get the integral equation satisfied by the expected discounted dividend payments

$$
\begin{aligned}
\phi(u)= & \int_{0}^{\infty}\left[\phi(u+y) I_{\left\{u+y \leq b_{2}\right\}}+\left(\chi_{2}\left(u+y-b_{2}\right)\right)+\phi\left(b_{2}\right) I_{\left\{u+y>b_{2}\right\}}\right] g_{\delta,-}(y) d y \\
& +\int_{0}^{u}\left[\phi\left(b_{1}\right) I_{\left\{u-y \leq b_{1}\right\}}+\phi(x-y) I_{\left\{u-y>b_{1}\right\}}\right] g_{\delta,+}(y) d y .
\end{aligned}
$$

Consider the following integral:

$$
\int_{0}^{\infty} \chi_{2}\left(u+y-b_{2}\right) I_{\left\{u+y>b_{2}\right\}} g_{\delta,-}(y) d y=\int_{b_{2}-u}^{\infty} \chi_{2}\left(u+y-b_{2}\right) g_{\delta,-}(y) d y,
$$

and let $z=(u+y)-b_{2}$, then $y=z+b_{2}-u$. After bringing $g_{\delta,-}(y)$ into (37), the above integral can be simplified as follows:

$$
\begin{aligned}
& \int_{b_{2}-u}^{\infty} \chi_{2}\left(u+y-b_{2}\right) g_{\delta,-}(y) d y \\
&=\int_{0}^{\infty} \chi_{2}(z) g_{\delta,-}\left(z+b_{2}-u\right) d z \\
&=\sum_{j=1}^{n} B_{j}^{*} \int_{0}^{\infty} \chi_{2}(z) \frac{\left(z+b_{2}-u\right)^{j-1}}{(j-1) !} e^{-\rho_{\gamma}\left(z+b_{2}-u\right)} d z \\
&=\sum_{j=1}^{n} B_{j}^{*} e^{\rho_{\gamma}\left(u-b_{2}\right)} \int_{0}^{\infty} \chi_{2}(z) \frac{\left(z+\left(b_{2}-u\right)\right)^{j-1}}{(j-1) !} e^{-\rho_{\gamma} z} d z \\
&=\sum_{j=1}^{n} B_{j}^{*} e^{\rho_{\gamma}\left(u-b_{2}\right)} \int_{0}^{\infty} \chi_{2}(z) \sum_{l=1}^{j} \frac{\left(b_{2}-u\right)^{l-1} z^{j-1}}{(l-1) !(j-1) !} e^{-\rho_{\gamma} z} d z \\
&=\sum_{j=1}^{n} B_{j}^{*} e^{\rho_{\gamma}\left(u-b_{2}\right)} \sum_{l=1}^{j} \frac{\left(b_{2}-u\right)^{l-1}}{(l-1) !} \int_{0}^{\infty} \chi_{2}(z) \sum_{l=1}^{j} \frac{z^{j-l}}{(j-l) !} e^{-\rho_{\gamma} z} d z \\
&=\sum_{j=1}^{n} B_{j}^{*} e^{\rho_{\gamma}\left(u-b_{2}\right)} \sum_{l=1}^{j} \sum_{m=1}^{l} \frac{(-u)^{m-1} b_{2}^{l-m}}{(m-1) !(l-m) !} \int_{0}^{\infty} \chi_{2}(z) \frac{z^{j-l}}{(j-l) !} e^{-\rho_{\gamma} z} d z \\
&= \sum_{m=1}^{n} \sum_{l=m}^{n} \sum_{j=l}^{n} B_{j}^{*} e^{-\rho_{\gamma} b_{2}} \frac{b_{2}^{l-m}}{(l-m) !}(-1)^{m-1} \frac{u^{m-1}}{(m-1) !} e^{\rho_{\gamma} u} \\
& \times \int_{0}^{\infty} \chi_{2}(z) \frac{z^{j-l}}{(j-l) !} e^{-\rho_{\gamma} z} d z .
\end{aligned}
$$

The operations with the other integrals are exactly the same as that of the related integral in the Gerber-Shiu function. By applying the operator $\left(\frac{d}{d u}-\rho_{\gamma}\right)^{n} \prod_{i=1}^{r}\left(\frac{d}{d u}+k_{i}\right)^{n}$ on both sides of Eq. (36) at the same time, a higher-order differential equation on $\varphi(u)$ can be obtained. The general solution to this equation can be obtained as follows:

$$
\phi(u)=\sum_{z=1}^{n(r+1)} D_{z} e^{\alpha_{z} u}
$$


where $\alpha_{Z}$ is also the characteristic root corresponding to the above higher-order differential equation. Substitute formula (39) into Eq. (36), and calculate the two integrals on the right. The first integral in Eq. (36) can be directly obtained by using the result of the related integral in Eq. (15)

$$
\begin{aligned}
\int_{0}^{\infty}[ & \left.\phi(u+y) I_{\left\{x+y \leq b_{2}\right\}}+\left(\phi\left(b_{2}\right)+\chi_{2}\left(u+y-b_{2}\right)\right) I_{\left\{u+y>b_{2}\right\}}\right] g_{\delta,-}(y) d y \\
= & \int_{0}^{b_{2}-u} \phi(u+y) g_{\delta,-}(y) d y+\int_{b_{2}-u}^{\infty}\left(\phi\left(b_{2}\right)+\chi_{2}\left(u+y-b_{2}\right)\right) g_{\delta,-}(y) d y \\
= & \sum_{z=1}^{n(r+1)} D_{z} e^{\alpha_{z} u} \sum_{j=1}^{n} B_{j}^{*} \frac{1}{\left(\rho_{\gamma}-\alpha_{z}\right)^{j}} \\
& -\sum_{z=1}^{n(r+1)} D_{z} \sum_{m=1}^{n} \sum_{l=m}^{n} \sum_{j=l}^{n} B_{j}^{*} \frac{1}{\left(\rho_{\gamma}-\alpha_{z}\right)^{j+1-l}} \frac{b_{2}^{l-m} e^{\left(\alpha_{z}-\rho_{\gamma}\right) b_{2}}}{(l-m) !} \frac{(-1)^{m-1} u^{m-1}}{(m-1) !} e^{\rho_{\gamma} u} \\
& +\sum_{z=1}^{n(r+1)} D_{z} \sum_{m=1}^{n} \sum_{l=m}^{n} \sum_{j=l}^{n} B_{j}^{*} \frac{1}{\rho_{\gamma}^{j+1-l}} \frac{b_{2}^{l-m} e^{\left(\alpha_{z}-\rho_{\gamma}\right) b_{2}}}{(l-1)^{m-1} u^{m-1}} e^{\rho_{\gamma} u} \frac{(m-1) !}{(m)} \\
& +\sum_{m=1}^{n} \sum_{l=m}^{n} \sum_{j=l}^{n} B_{j}^{*} e^{-\rho_{\gamma} b_{2}} \frac{b_{2}^{l-m}}{(l-m) !} \frac{(-1)^{m-1} u^{m-1}}{(m-1) !} e^{-\rho_{\gamma} u} \int_{0}^{\infty} \chi_{2}(z) \frac{z^{j-l}}{(j-l) !} e^{-\rho_{\gamma} z} d z \\
& \sum_{z=1}^{n(r+1)} D_{z} e^{\alpha_{z} u} \sum_{j=1}^{n} B_{j}^{*} \frac{1}{\left(\rho_{\gamma}-\alpha_{z}\right)^{j}} \\
& \quad \times \int_{0}^{n(r+1)} \chi_{2}(z) \frac{z^{j-l}}{(j-l) !} e^{-\rho_{\gamma} z} d z . \\
& +\sum_{z=1}^{n} D_{z} \sum_{m=1}^{n} \sum_{l=m}^{n} \sum_{j=l}^{n} B_{j}^{*}\left(\frac{1}{\rho_{\gamma}^{j+1-l}}-\frac{1}{\left(\rho_{\gamma}-\alpha_{z}\right)^{j+1-l}}\right) \\
& \times \frac{b_{2}^{l-m} e^{\left(\alpha_{z}-\rho_{\gamma}\right) b_{2}}}{(l-m) !} \frac{(-1)^{m-1} u^{m-1}}{(m-1) !} e^{\rho_{\gamma} u} \\
& +\sum_{m=1}^{n} \sum_{l=m}^{n} \sum_{j=l}^{n} B_{j}^{*} e^{-\rho_{\gamma} b_{2}} \frac{b_{2}^{l-m}}{(l-m) !} \frac{(-1)^{m-1} u^{m-1}}{(m-1) !} e^{-\rho_{\gamma} u} \\
&
\end{aligned}
$$

By Eq. (15), the second integral of Eq. (36) is calculated as follows:

$$
\begin{aligned}
\int_{0}^{\infty} & {\left[\phi\left(b_{1}\right) I_{\left\{u-y \leq b_{1}\right\}}+\phi(u-y) I_{\left\{u-y>b_{1}\right\}}\right] g_{\delta,+}(y) d y } \\
= & \int_{u-b_{1}}^{u} \phi\left(b_{1}\right) g_{\delta,+}(y) d y+\int_{0}^{u-b_{1}} \phi(u-y) g_{\delta,+}(y) d y \\
& \quad+\sum_{z=1}^{n(r+1)} D_{z} \sum_{i=1}^{r} \sum_{m=1}^{n}\left[\sum_{l=m}^{n} \sum_{j=l}^{n} B_{i j} \frac{\left(-b_{1}\right)^{l-m} e^{\left(k_{i}+\alpha_{z}\right) b_{1}}}{k_{i}^{j+1-l}(l-m) !}-\sum_{j=m}^{n} B_{i j} \frac{e^{\alpha_{z} b_{1}}}{k_{i}^{j+1-l}}\right] \frac{u^{m-1}}{(m-1) !} e^{-k_{i} u} \\
& +\sum_{z=1}^{n(r+1)} D_{z} e^{\alpha_{z} u} \sum_{i=1}^{r} \sum_{j=1}^{n} B_{i j} \frac{1}{\left(k_{i}+\alpha_{z}\right)^{j}}
\end{aligned}
$$




$$
\begin{aligned}
& -\sum_{z=1}^{n(r+1)} D_{z} \sum_{i=1}^{r} \sum_{m=1}^{n} \sum_{l=m}^{n} \sum_{j=l}^{n} B_{i j} \frac{1}{\left(k_{i}+\alpha_{z}\right)^{j+1-l}} \frac{\left(-b_{1}\right)^{l-m}}{(l-m) !} e^{\left(k_{i}+\alpha_{z}\right) b_{1}} \frac{u^{m-1}}{(m-1) !} e^{-k_{i} u} \\
= & \sum_{z=1}^{n(r+1)} D_{z} e^{\alpha_{z} u} \sum_{i=1}^{r} \sum_{j=1}^{n} B_{i j} \frac{1}{\left(k_{i}+\alpha_{z}\right)^{j}} \\
& +\sum_{z=1}^{n(r+1)} D_{z} \sum_{i=1}^{r} \sum_{m=1}^{n}\left[\sum_{l=m}^{n} \sum_{j=l}^{n} B_{i j}\left(\frac{1}{k_{i}^{j+1-l}}-\frac{1}{\left(k_{i}+\alpha_{z}\right)^{j+1-l}}\right) e^{\left(k_{i}+\alpha_{z}\right) b_{1}} \frac{\left(-b_{1}\right)^{l-m}}{(l-m) !}\right. \\
& \left.-\sum_{j=m}^{n} B_{i j} \frac{1}{k_{i}^{j+1-l}} e^{\alpha_{z} b_{1}}\right] \frac{u^{m-1}}{(m-1) !} e^{-k_{i} u} .
\end{aligned}
$$

Substituting the two integrals (40) and (41) into formula (39), we have

$$
\begin{aligned}
& \phi(u)=\sum_{z=1}^{n(r+1)} D_{z} e^{\alpha_{z} u} \\
& =\sum_{z=1}^{n(r+1)} D_{z} e^{\alpha_{z} u} \sum_{j=1}^{n} B_{j}^{*} \frac{1}{\left(\rho_{\gamma}-\alpha_{z}\right)^{j}}+\int_{b_{2}-x}^{\infty} \chi_{2}\left(u+y-b_{2}\right) g_{\delta,-}(y) d y \\
& +\sum_{z=1}^{n(r+1)} D_{z} \sum_{m=1}^{n} \sum_{l=m}^{n} \sum_{j=l}^{n} B_{j}^{*}\left(\frac{1}{\rho_{\gamma}^{j+1-l}}-\frac{1}{\left(\rho_{\gamma}-\alpha_{z}\right)^{j+1-l}}\right) \\
& \times \frac{b_{2}^{l-m} e^{\left(\alpha_{z}-\rho_{\gamma}\right) b_{2}}}{(l-m) !} \frac{(-1)^{m-1} u^{m-1}}{(m-1) !} e^{\rho_{\gamma} u} \\
& +\sum_{z=1}^{n(r+1)} D_{z} \sum_{i=1}^{r} \sum_{m=1}^{n}\left[\sum_{l=m}^{n} \sum_{j=l}^{n} B_{i j}\left(\frac{1}{k_{i}^{j+1-l}}-\frac{1}{\left(k_{i}+\alpha_{z}\right)^{j+1-l}}\right) e^{\left(k_{i}+\alpha_{z}\right) b_{1}} \frac{\left(-b_{1}\right)^{l-m}}{(l-m) !}\right. \\
& \left.-\sum_{j=m}^{n} B_{i j} \frac{1}{k_{i}^{j+1-l}} e^{\alpha_{z} b_{1}}\right] \frac{u^{m-1}}{(m-1) !} e^{-k_{i} u}+\sum_{z=1}^{n(r+1)} D_{z} e^{\alpha_{z} u} \sum_{i=1}^{r} \sum_{j=1}^{n} B_{i j} \frac{1}{\left(k_{i}+\alpha_{z}\right)^{j}} \\
& =\sum_{z=1}^{n(r+1)} D_{z} e^{\alpha_{z} u}\left[\sum_{j=1}^{n} B_{j}^{*} \frac{1}{\left(\rho_{\gamma}-\alpha_{z}\right)^{j}}+\sum_{i=1}^{r} \sum_{j=1}^{n} B_{i j} \frac{1}{\left(k_{i}+\alpha_{z}\right)^{j}}\right] \\
& +\sum_{z=1}^{n(r+1)} D_{z} \sum_{m=1}^{n} \sum_{l=m}^{n} \sum_{j=l}^{n} B_{j}^{*}\left(\frac{1}{\rho_{\gamma}^{j+1-l}}-\frac{1}{\left(\rho_{\gamma}-\alpha_{z}\right)^{j+1-l}}\right) \\
& \times \frac{b_{2}^{l-m} e^{\left(\alpha_{z}-\rho_{\gamma}\right) b_{2}}}{(l-m) !} \frac{(-1)^{m-1} u^{m-1}}{(m-1) !} e^{\rho_{\gamma} u} \\
& +\sum_{z=1}^{n(r+1)} D_{z} \sum_{i=1}^{r} \sum_{m=1}^{n}\left[\sum_{l=m}^{n} \sum_{j=l}^{n} B_{i j}\left(\frac{1}{k_{i}^{j+1-l}}-\frac{1}{\left(k_{i}+\alpha_{z}\right)^{j+1-l}}\right)\right. \\
& \times e^{\left(k_{i}+\alpha_{z}\right) b_{1}} \frac{\left(-b_{1}\right)^{l-m}}{(l-m) !} \\
& \left.-\sum_{j=m}^{n} B_{i j} \frac{1}{k_{i}^{j+1-l}} e^{\alpha_{z} b_{1}}\right] \frac{u^{m-1}}{(m-1) !} e^{-k_{i} u}
\end{aligned}
$$




$$
\begin{aligned}
& +\sum_{m=1}^{n} \sum_{l=m}^{n} \sum_{j=l}^{n} B_{j}^{*} e^{-\rho_{\gamma} b_{2}} \frac{b_{2}^{l-m}}{(l-m) !}(-1)^{m-1} \frac{u^{m-1}}{(m-1) !} e^{-\rho_{\gamma} u} \\
& \times \int_{0}^{\infty} \chi_{2}(z) \frac{z^{j-l}}{(j-l) !} e^{-\rho_{\gamma} z} d z .
\end{aligned}
$$

The result of the above integral will depend on the form of the loss function $\chi_{2}(x)$. When the form of function $\chi_{2}(x)$ is given, the specific result of the above integral can be calculated. It is consistent with the solution method for the Gerber-Shiu function. After combining similar terms, the equation satisfied by $n(r+1)$ coefficients can be obtained according to the above result. Therefore, all parameters contained in $\phi(u)=\sum_{z=1}^{n(r+1)} D_{z} e^{\alpha_{z} u}$ can be found, and then the expression of $\phi(u)$ can be obtained.

\section{Numerical illustrations}

In this section, we give some examples of the Gerber-Shiu function, the expected discounted capital injection, and the expected discounted dividend payments.

Example 1 It is assumed that the interobservation time is $\operatorname{Erlang}(2,2)$-distributed, the arrival time of a claim and the amount of a single claim are exponentially distributed with parameters $\lambda=1, v=1$, respectively. The premium charged per unit time is assumed to be $c=2$ and the penalty function is $\omega(x)=1$. Now we consider the influences of interest force $\delta$, injection line $b_{1}$, dividend payments line $b_{2}$ on the Laplace transformation of ruin time, the expected discounted capital injection until ruin, and the expected discounted dividend payments until ruin separately.

As can be seen in Fig. 2, the Laplace transformation of ruin time is a decreasing function of initial surplus $u$, which is contrary to the conclusion of traditional actuarial model. This shows that a higher initial surplus $u$ leads to a smaller Laplace transformation of the ruin time. This is because the function $e^{-\delta \tau_{b_{1}}^{b_{2}}}$ is a decreasing function of ruin time $\tau_{b_{1}}^{b_{2}}$. The larger initial surplus $u$ leads to a larger ruin time $\tau_{b_{1}}^{b_{2}}$, and a smaller Laplace transformation of ruin time is obtained due to the decreasing function $e^{-\delta \tau_{b_{1}}^{b_{2}}}$. Moreover, when the initial surplus $u$ is fixed, the Laplace transformation of ruin time is a decreasing function for parameters $\delta, b_{1}$, and $b_{2}$, respectively.

In Fig. 3, we see that the expected discounted capital injection until ruin is also a decreasing function of the initial surplus $u$. When the initial surplus $u$ is fixed, the expected discounted capital injection until ruin is a decreasing function of parameters $\delta$ and $b_{2}$, respectively, and an increasing function of $b_{1}$.

In Fig. 4, we see that the expected discounted dividend payments until ruin is an increasing function of the initial surplus $u$. When the initial surplus $u$ is fixed, the expected discounted dividend payments until ruin is a decreasing function of the parameters $\delta$ and $b_{2}$, respectively, and an increasing function of $b_{1}$.

Next, we will analyze the influence on the Laplace transformation of ruin time, the expected discounted capital injection until ruin, and the expected discounted dividend payments until ruin when the single claim amount is subject to the following four distributions:

(1) Exponential distribution $(\operatorname{Exp}) f_{Y}(y)=e^{-y}$;

(2) Combined exponential distribution (Com-Exp) $f_{Y}(y)=2 \times 1.5 e^{-1.5 y}-3 e^{-3 y}$; 


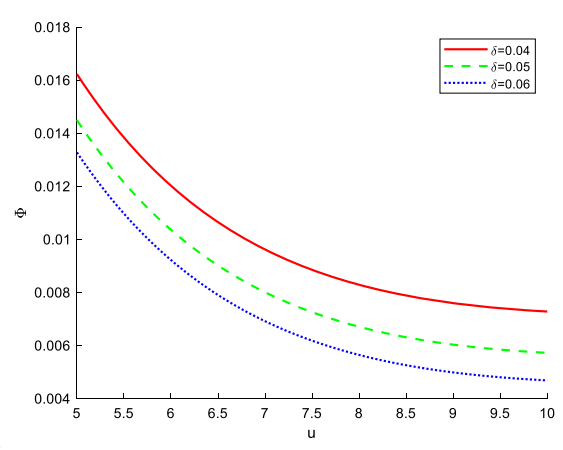

(a) $b_{1}=5 ; b_{2}=10$

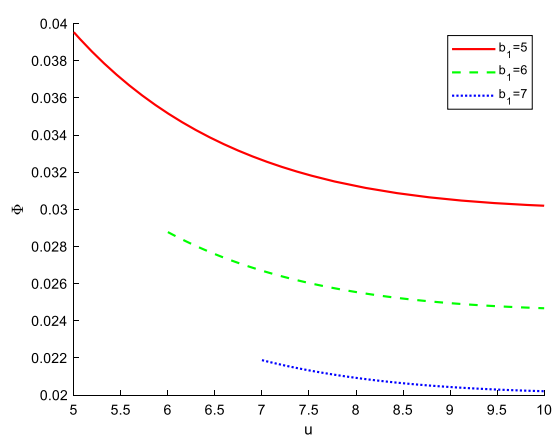

(b) $\delta=0.01 ; b_{2}=10$

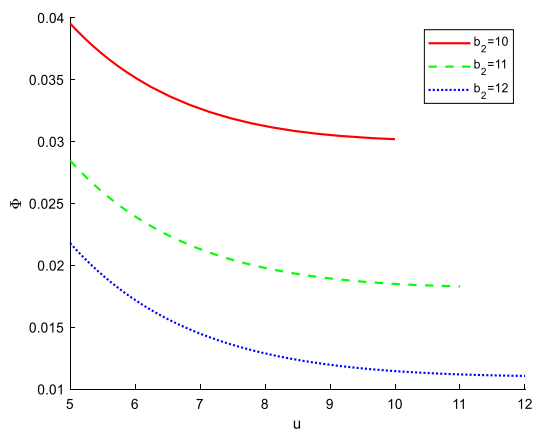

(c) $\delta=0.01 ; b_{1}=5$

Figure 2 The Laplace transformation of ruin time

(3) Mixed exponential distribution (Mix-Exp) $f_{Y}(y)=\frac{1}{3} \times 2 e^{-2 y}+\frac{2}{3} \times 0.8 e^{-0.8 y}$;

(4) $\operatorname{Erlang}(2,2)$ distribution $f_{Y}(y)=4 y e^{-2 y}$.

Example 2 It is assumed that the interobservation time is $\operatorname{Erlang}(2,2)$-distributed, the arrival time of claim is exponentially distributed with parameters $\lambda=1$. Let $c=1.5, \delta=0.01$, $b_{1}=5$, and $b_{2}=10$. We consider the influence of the above four probability distributions of a single claim amount on the Laplace transformation of the ruin time.

As one can see in Fig. 5, the Laplace transformation of the ruin time is a decreasing function of the initial surplus $u$, and it is easy to see that when the average value of claims is equal, the Laplace transformation of the ruin time will increase with the increase of the variance of the claim amount distribution.

Example 3 It is assumed that the interobservation time is $\operatorname{Erlang}(2,2)$-distributed, the arrival time of claim is exponentially distributed with parameters $\lambda=1$. Let $c=5, \delta=0.01$, $b_{1}=2$, and $b_{2}=6$. We consider the influence of the above four probability distributions of a single claim amount on the expected discounted capital injection until ruin.

It can be concluded from Fig. 6 that the expected discounted capital injection until ruin is no longer a strictly decreasing function of the initial surplus $u$, and its monotonicity will change with the different distribution of claims. When the claim amount follows the exponential distribution and mixed exponential distribution, the expected discounted capital injection until ruin decreases strictly monotonically with respect to the initial surplus $u$. 


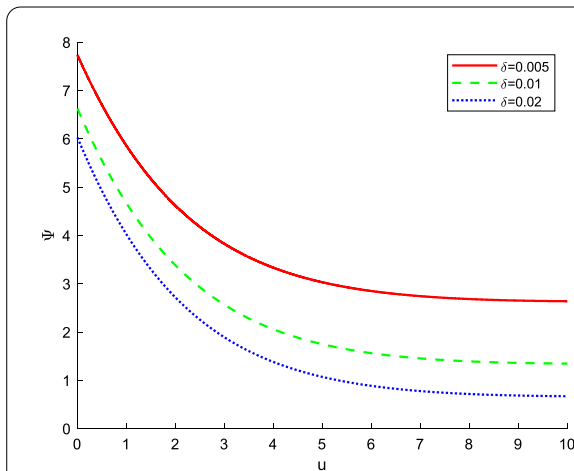

a: $b_{1}=5 ; b_{2}=10$

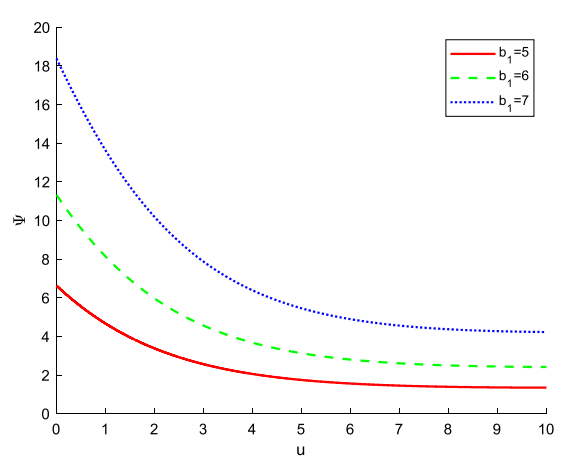

b: $\delta=0.01 ; b_{2}=10$

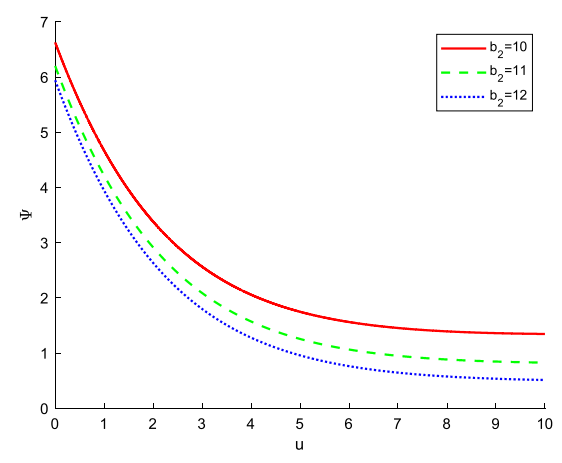

c: $\delta=0.01 ; b_{1}=5$

Figure 3 The expected discounted capital injection until ruin

When the claim amount follows the combined exponential distribution and Erlang distribution, the expected discounted capital injection function will first increase with the increase of the initial surplus $u$, and then decrease with the increase of the initial surplus $u$ after passing a certain special value. And when the initial surplus $u$ exceeds a special value, the expected discounted capital injection function until ruin will increase with the increase of the variance of the claim amount distribution.

Example 4 It is assumed that the interobservation time is $\operatorname{Erlang}(2,2)$-distributed, the arrival time of a claim is exponentially distributed with parameters $\lambda=1$. Let $c=5$, $\delta=0.01, b_{1}=2$, and $b_{2}=6$. We consider the influence of the above four probability distributions of a single claim amount on the expected discounted dividend payments until ruin.

Here one can see from Fig. 7 that the expected discounted dividend payments until ruin is an increasing function of the initial surplus $u$. And it is easy to see that when the average value of the claim amount distribution is equal, the expected discounted dividend payments until ruin will decrease with the increase of the variance of the claim amount distribution. When the claim amount distribution is Erlang, the expected discounted dividend payments until ruin is the largest, and when the claim amount distribution is a mixed exponential distribution, the expected discounted dividend payments until ruin are the smallest. 


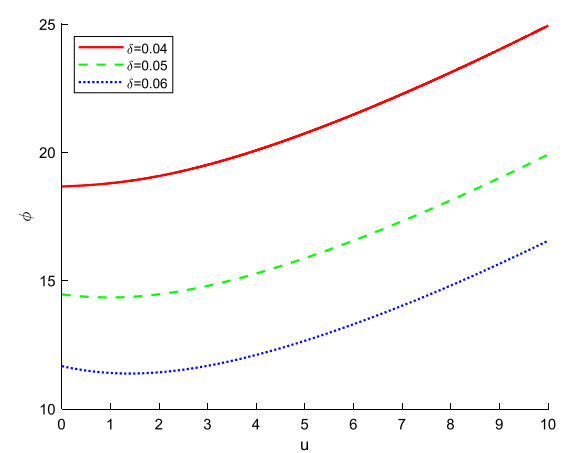

a: $b_{1}=5 ; b_{2}=10$

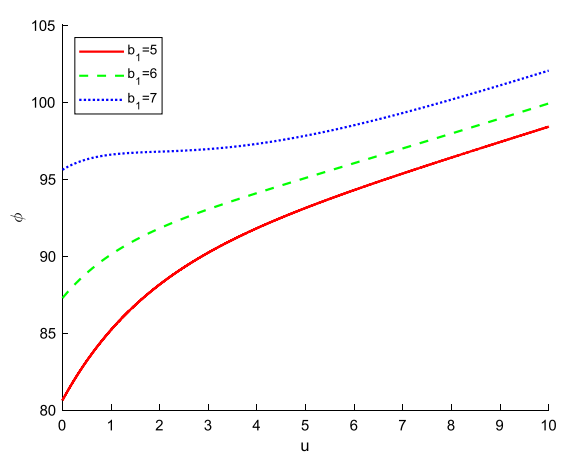

b: $\delta=0.01 ; b_{2}=10$

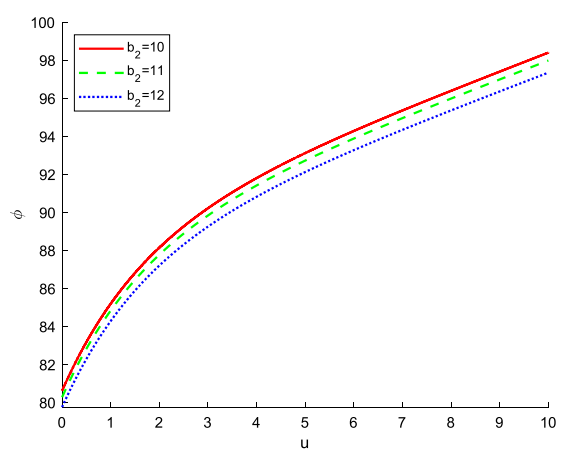

c: $\delta=0.01 ; b_{1}=5$

Figure 4 The expected discounted dividend payments until ruin

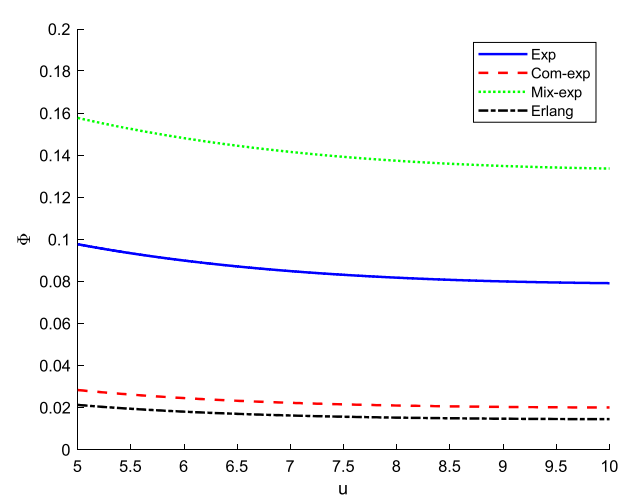

Figure 5 The Laplace transformation of the ruin time

However, it is worth noting that the injection and dividend levels in the model are assumed in advance, which are not necessarily the optimal injection and dividend levels. So later, the topic can also focus on the selection of the optimal capital injection and dividend levels. 


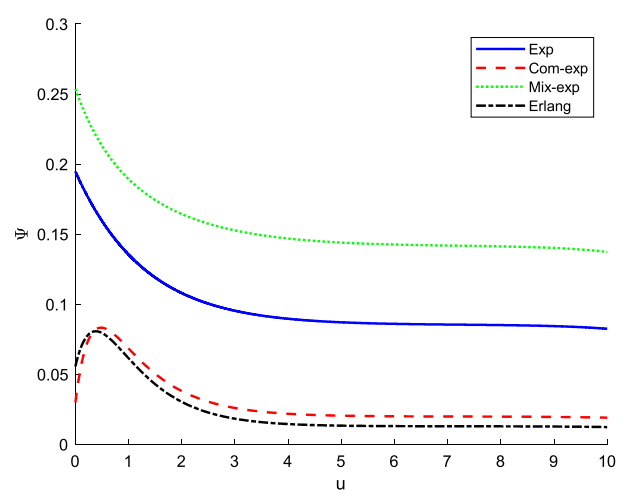

Figure 6 The expected discounted capital injection until ruin

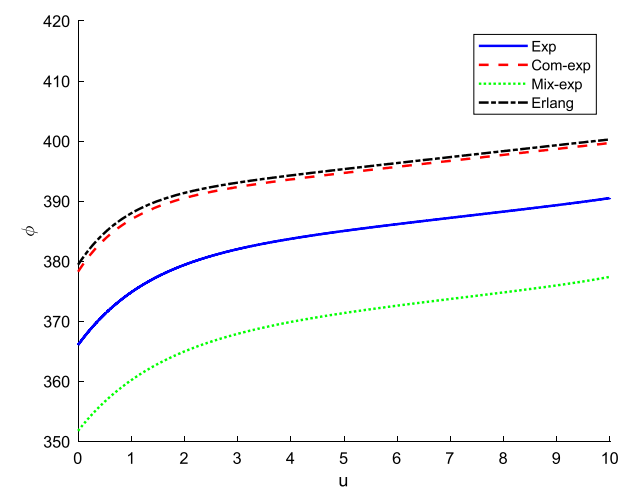

Figure 7 The expected discounted dividend payments until ruin

\section{Acknowledgements}

The authors thank the referees for a number of suggestions, which have improved many aspects of this paper.

\section{Funding}

This research was supported by the National Natural Science Foundation of China (Grant Nos. 11301303), the Taishan Scholars Program of Shandong Province (Grant No. tsqn20161041), the Humanities and Social Sciences Project of the Ministry Education of China (Grant No. 19YJA910002), the Natural Science Foundation of Shandong Province (Grant No. ZR2018MG002), the Fostering Project of Dominant Discipline and Talent Team of Shandong Province Higher Education Institutions (Grant No. 1716009), the Shandong Provincial Social Science Project Planning Research Project (Grant No. 19CQXJ08), the 1251 Talent Cultivation Project of Shandong Jiaotong University, the Risk Management and Insurance Research Team of Shandong University of Finance and Economics, the Shandong Jiaotong University 'Climbing' Research Innovation Team Program, and the Collaborative Innovation Center Project of the Transformation of New and Old Kinetic Energy and Government Financial Allocation, Excellent Talents Project of Shandong University of Finance and Economics.

\section{Availability of data and materials}

Please contact authors for data requests.

\section{Competing interests}

The authors declare that they have no competing interests.

\section{Authors' contributions}

All authors contributed equally to the writing of this paper. The authors read and approved the final manuscript.

\section{Author details}

${ }^{1}$ School of Insurance, Shandong University of Finance and Economics, Jinan 250014, P.R. China. ${ }^{2}$ College of Mathematics and Statistics, Chongqing University, Chongqing 401331, P.R. China. ${ }^{3}$ School of Mathematic and Quantitative Economics, Shandong University of Finance and Economics, Jinan 250014, P.R. China. ${ }^{4}$ School of Science, Shandong Jiaotong University, Jinan 250357, P.R. China. 


\section{Publisher's Note}

Springer Nature remains neutral with regard to jurisdictional claims in published maps and institutional affiliations.

\section{Received: 10 June 2020 Accepted: 14 April 2021 Published online: 26 April 2021}

\section{References}

1. Lundberg, F: Approximerad Framställning av Sannolikehetsfunktionen, Aterförsäkering av Kollektivrisker. Almqvist \& Wiksell, Uppsala. Akad. Afhandling. Almqvist \& Wiksell, Uppsala (1903)

2. Gerber, H.U., Shiu, E.S.W.: On optimal dividend strategies in the compound Poisson model. N. Am. Actuar. J. 10(2), 76-93 (2006)

3. Chi, Y.C., Lin, X.S.: On the threshold dividend strategy for a generalized jump-diffusion risk model. Insur. Math. Econ. $48(3), 326-337(2011)$

4. Yu, W.G.: Some results on absolute ruin in the perturbed insurance risk model with investment and debit interests. Econ. Model. 31, 625-634 (2013)

5. Yin, C.C., Wen, Y.Z., Zhao, Y.X.: On the optimal dividend problem for a spectrally positive Lévy process. ASTIN Bull. 44(3), 635-651 (2014)

6. Shen, Y., Yin, C.C., Yuen, K.C.: Alternative approach to the optimality of the threshold strategy for spectrally negative Lévy processes. Acta Math. Appl. Sin. Engl. Ser. 29, 705-716 (2013)

7. Yu, W.G., Huang, Y.J., Cui, C.R.: The absolute ruin insurance risk model with a threshold dividend strategy. Symmetry 10(9), $377(2018)$

8. Yu, W.G., Yong, Y.D., Guan, G.F., Huang, Y.J., Su, W., Cui, C.R.: Valuing guaranteed minimum death benefits by cosine series expansion. Mathematics 7(9), 835 (2019)

9. Zhou, M., Yuen, K.C., Yin, C.C.: Optimal investment and premium control in a nonlinear diffusion model. Acta Math. Appl. Sin. Engl. Ser. 33, 945-958 (2017)

10. Zhou, J.M., Mo, X.Y., Ou, H., Yang, X.Q.: Expected present value of total dividends in the compound binomial model with delayed claims and random income. Acta Math. Sci. 33(6), 1639-1651 (2013)

11. Xu, L., Yao, D.J., Cheng, G.P.: Optimal investment and dividend for an insurer under a Markov regime switching market with high gain tax. J. Ind. Manag. Optim. 16(1), 325-356 (2020)

12. Yin, C.C., Wen, Y.Z:: Optimal dividend problem with a terminal value for spectrally positive Lévy processes. Insur. Math. Econ. 53(3), 769-773 (2013)

13. Dong, H., Yin, C.C., Dai, H.S.: Spectrally negative Lévy risk model under Erlangized barrier strategy. J. Comput. Appl. Math. 351, 101-116 (2019)

14. Li, D.P., Rong, X.M., Zhao, H.: Optimal reinsurance and investment problem for an insurer and a reinsurer with jump-diffusion risk process under the Heston model. Comput. Appl. Math. 35, 533-557 (2016)

15. Peng, J.Y., Wang, D.C.: Uniform asymptotics for ruin probabilities in a dependent renewal risk model with stochastic return on investments. Stochastics 90(3), 432-471 (2018)

16. Yao, D.J., Wang, R.M., Xu, L.: Optimal impulse control for dividend and capital injection with proportional reinsurance and exponential premium principle. Commun. Stat., Theory Methods 46(5), 2519-2541 (2017)

17. He, L., Liang, Z.X.: Optimal financing and dividend control of the insurance company with fixed and proportional transaction costs. Insur. Math. Econ. 44(1), 88-94 (2009)

18. Zhu, J.X., Yang, H.L.: Optimal capital injection and dividend distribution for growth restricted diffusion models with bankruptcy. Insur. Math. Econ. 70, 259-271 (2016)

19. Albrecher, H., Cheung, E.C.K., Thonhauser, S.: Randomized observation periods for the compound Poisson risk model: dividends. ASTIN Bull. 41(2), 645-672 (2011)

20. Avanzi, B., Cheung, E.C.K., Wong, B., Woo, J.K.: On a periodic dividend barrier strategy in the dual model with continuous monitoring of solvency. Insur. Math. Econ. 52(1), 98-113 (2013)

21. Zhao, Y.X., Chen, P., Yang, H.L.: Optimal periodic dividend and capital injection problem for spectrally positive Lévy processes. Insur. Math. Econ. 74, 135-146 (2017)

22. Zhang, Z.M., Cheung, E.C.K., Yang, H.L.: On the compound Poisson risk model with periodic capital injections. ASTIN Bull. 48(1), 435-477 (2018)

23. Cheung, E.C.K., Zhang, Z.M.: Periodic threshold type dividend strategy in the compound Poisson risk model. Scand. Actuar. J. 2019(1), 1-31 (2019)

24. Peng, X.H., Su, W., Zhang, Z.M.: On a perturbed compound Poisson risk model under a periodic threshold-type dividend strategy. J. Ind. Manag. Optim. 16(4), 1967-1986 (2020)

25. Pérez, J.L., Yamazaki, K.: On the optimality of periodic barrier strategies for a spectrally positive Lévy process. Insur. Math. Econ. 77, 1-13 (2017)

26. Noba, K., Pérez, J.L., Yamazaki, K., Yano, K.: On optimal periodic dividend strategies for Lévy risk processes. Insur. Math. Econ. 80, 29-44 (2018)

27. Yang, L., Deng, G.H.: A perturbed risk model with constant interest and periodic barrier dividend strategy. Commun. Stat., Simul. Comput. 1614620 (2019)

28. Dong, H., Zhou, X.W.: On a spectrally negative Lévy risk process with periodic dividends and capital injections. Stat. Probab. Lett. 155, 108589 (2019)

29. Dong, H., Zhao, X.H.: On periodic dividends for the classical risk model with debit interest. Math. Probl. Eng. 2020, $6395717(2020)$

30. Yang, X.X., Tan, J.Y., Zhang, H.J., Li, Z.Q.: An optimal control problem in a risk model with stochastic premiums and periodic dividend payments. Asia-Pac. J. Oper. Res. 34(3), 1740013 (2017)

31. Liu, Y.G., Chen, X., Zhuo, W.Y.: Dividends under threshold dividend strategy with randomized observation periods and capital-exchange agreement. J. Comput. Appl. Math. 366, 112426 (2020)

32. Yu, W.G., Guo, P., Wang, Q., Guan, G.F., Yang, Q., Huang, Y.J., Yu, X.L., Jin, B.Y., Cui, C.R.: On a periodic capital injection and barrier dividend strategy in the compound Poisson risk model. Mathematics 8(4), 511 (2020)

33. Gerber, H.U., Shiu, E.S.W.: On the time value of ruin. N. Am. Actuar. J. 2(1), $48-78$ (1998)

34. Lin, X.S., Willmot, G.E., Drekic, S.: The classical risk model with a constant dividend barrier: analysis of the Gerber-Shiu discounted penalty function. Insur. Math. Econ. 33(3), 551-566 (2003) 
35. Willmot, G.E., Dickson, D.C.M.: The Gerber-Shiu discounted penalty function in the stationary renewal risk model. Insur. Math. Econ. 32(3), 403-411 (2003)

36. Li, S.M., Lu, Y., Sendova, K.P.: The expected discounted penalty function: from infinite time to finite time. Scand. Actuar. J. 2019(4), 336-354 (2019)

37. Huang, Y.J., Yu, W.G., Pan, Y., Cui, C.R.: Estimating the Gerber-Shiu expected discounted penalty function for Lévy risk model. Discrete Dyn. Nat. Soc. 2019, 3607201 (2019)

38. Zhang, Z.M., Su, W.: Estimating the Gerber-Shiu function in a Lévy risk model by Laguerre series expansion. J. Comput. Appl. Math. 346, 133-149 (2019)

39. Preischl, M., Thonhauser, S.: Optimal reinsurance for Gerber-Shiu functions in the Cramér-Lundberg model. Insur. Math. Econ. 87, 82-91 (2019)

40. Zhang, Z.M., Yong, Y.D., Yu, W.G.: Valuing equity-linked death benefits in general exponential Lévy models. J. Comput. Appl. Math. 365, 112377 (2020)

41. Palmowski, Z., Vatamidou, E.: Phase-type approximations perturbed by a heavy-tailed component for the Gerber-Shiu function of risk processes with two-sided jumps. Stoch. Models 36(2), 337-363 (2020)

42. Albrecher, H., Cheung, E.C.K. Thonhauser, S.: Randomized observation periods for the compound Poisson risk model: the discounted penalty function. Scand. Actuar. J. 2013(6), 424-452 (2013)

\section{Submit your manuscript to a SpringerOpen ${ }^{\circ}$ journal and benefit from:}

- Convenient online submission

- Rigorous peer review

- Open access: articles freely available online

- High visibility within the field

- Retaining the copyright to your article

Submit your next manuscript at $\boldsymbol{\nabla}$ springeropen.com 